\title{
Corrected Horváth-Kawazoe Equations for Pore-Size Distribution
}

\author{
Salil U. Rege and Ralph T. Yang \\ Dept. of Chemical E ngineering, U niversity of M ichigan, A nn A rbor, MI 48109
}

\begin{abstract}
The Horváth-Kawazoe (HK) model is a widely used method for determining pore-size distribution in a microporous material from a single adsorption isotherm. The original model, however, suffers from conceptual flaws. The total interaction energy of the adsorbate is underestimated since the adsorbate-adsorbate interaction is incorrecty calculated. N ew corrected HK models proposed here for three pore geometries (slit, cylindrical, and spherical) can overcome these defects. Two other improvements have also been made in the new models. These assume that a filled micropore is composed of layers of adsorbate molecules which interact only with the molecular layers in the immediate $v$ icinity. A better estimate of adsorbate-adsorbate-adsorbent interaction is obtained by utilizing actual distances between interacting molecules. The average interaction energy is calculated by a population-weighted average of the energy potential of the layers rather than by integration. This average potential approaches a nonzero value at large pore size, unlike that in the original model. Pore-size distributions predicted by the corrected $\mathrm{HK}$ models agreed significantly better with crystallographic data compared to the original model for both microporous and mesoporous sorbents.
\end{abstract}

\section{Introduction}

The quantitative estimation of pore-size distribution (PSD) of materials remains a crucial problem in the characterization of microporous materials. Although several techniques exist for characterization of pore size of mesoporous and macroporous materials such as the Kelvin equation (B runauer et al., 1967), the BET equation (Brunauer et al., 1940), the BJH method (Barrett et al., 1951), and the DubininR adushkevich equation (Dubinin and R adushkevich, 1947), extension of these methods to microporous materials does not yield satisfactory results. This is primarily so because the adsorbate molecules can no longer be treated in a fluid state as the pore size approaches very low values (less than $20 \AA$ ), and the consideration of attractive forces on a molecular level becomes necessary. This problem is further coupled with the urgency for a simple and rapid technique for pore-size evaluation due to an increasing demand for new microporous materials for gas adsorption and catalysis. As a result, exhaustive research has been devoted to this area in recent times.

As an outcome of this research, several approaches have emerged with respect to the estimation of micropore-size dis-

Correspondence concerning this article should be addressed to R. T. Y ang tribution. O ne of the earliest methods was the experimental measurement of the adsorbed saturated amounts of probe molecules of different dimensions. This method was found to be cumbersome to perform and there was an uncertainty about the results due to the networking effects of pores such as a large pore accessible through small pores which are smaller than the probe molecule. A nother approach was to obtain the PSD from a single adsorption isotherm ( aroniec and Madey, 1988). The basis of this method involved an isotherm $w(L, P)$ dependent upon pore size $(L)$ and pressure (P). Thus, the amount adsorbed could be found by integrating the above isotherm over the entire range of pore size by coupling it with a PSD function $f(L)$. By assuming a known mathematical distribution function for $\mathrm{f}(\mathrm{L})$ and using an empirical relationship (Stoeckli, 1977) to obtain $w(L, P)$, it was possible to obtain the PSD function by solving for its parameters ( aroniec and M adey, 1988; $Y$ ang and Baksh, 1991; Baksh and $Y$ ang, 1992). However, despite its experimental simplicity, this procedure was undermined by the empiricism and assumptions involved. Based on these lines, recently new statistical mechanics approaches using either $\mathrm{M}$ onte $\mathrm{C}$ arlo techniques or the density functional theory have been proposed 
and are steadily gaining acceptance. These techniques enable the calculation of the isotherm (dependent on pore size and geometry) in conjunction with PSD functions with assumed mathematical forms. A large amount of literature has been published regarding the application of this theory for studying gas adsorption, and, in particular, evaluation of PSD of materials (Seaton et al., 1989; Lastoskie et al., 1993; Olivier, 1995; W ebb and Orr, 1997; R avikovitch et al., 1988). The advantage of the technique is that it is a unified method applicable to both microporous, as well as mesoporous type of materials. However, the empirical parameters used in these models are difficult to obtain from literature with the exception of a few well-studied systems. Besides, the calculations involved are highly intensive requiring an elaborate computer code.

A simple and popular method for evaluating PSD of microporous materials is the method proposed by Horváth and Kawazoe (1983). This technique has been successfully used for the determination of PSD in microporous sorbents such as activated carbons and zeolites over the last decade (Seifert and E mig, 1987; V enero and Chiou, 1988; D avis et al., 1988, 1989; Beck et al., 1992; Horváth et al., 1998; Kane et al., 1998). This technique utilizes adsorption isotherm data at a temperature below or equal to the critical temperature of the adsorbate, which is typically nitrogen (at $77 \mathrm{~K}$ ), argon (at 87 $\mathrm{K}$ ), or an organic vapor (such as $\mathrm{CH}_{3} \mathrm{Cl}$ ) at ambient temperature ( $\mathrm{M}$ ariwala and Foley, 1994). By assuming micropore-filling and equating the free-energy change upon adsorption to the average interaction energy of the adsorbing molecules, the "step" in the isotherm data is translated into a PSD. The model assumes that entropic effects are negligible for small adsorbate molecules and networking effects are insignificant if the adsorbate used in the analysis is smaller than the adsorbent channels. The mathematical expression relating relative pressure of the adsorbing gas to the pore size of the adsorbent has come to be known as the $\mathrm{H}$ orvath-K awazoe $(\mathrm{HK})$ equation.

The original HK equation considered a slit-shaped pore with an implicit assumption that the adsorption isotherm follows Henry' law. In order to correct for the deviation of isotherm data from Henry's law at higher relative pressures, Cheng and $Y$ ang (1994) proposed a correction factor, which incorporated a Langmuir isotherm fit to the data. A Iso, new models have been proposed based on the same principles to include pores with curved surfaces. Prominent among them are the cylindrical-pore model of Saito and Foley (1991) and the spherical pore model of Cheng and $Y$ ang (1994). Instruments incorporating these models have now been commercialized.

Despite the immense utility of the HK-model, there exist certain conceptual defects in the same. The derivation of the $\mathrm{HK}$-model proceeds by the calculation of the energy potential of a single adsorbate molecule with a layer of sorbent molecules along the periphery of a pore of a particular geometry using the Lennard-J ones 6-12 potential. This potential is calculated by incorporating the adsorbate-adsorbent dispersion term as calculated by the K irkwood-M üller formalism in the potential energy minimum $\epsilon^{*}$ (Horvath and Kawazoe, 1983). In order to include the adsorbate-adsorbate interactions, the adsorbate-adsorbate dispersion constant is appended to the adsorbate-adsorbent dispersion term in the potential energy minimum, as will be shown in detail later. However, no clear explanation is provided for doing so. Moreover, the energy potential due to adsorbate-adsorbate interactions in a filled pore can be interpreted as the energy profile due to the adsorbate molecules placed, rather unrealistically, at the same position as the adsorbate molecules. This is because, according to the original model, the distance $z$ of a sorbate molecule from another sorbate molecule is the same as that between a sorbate molecule and a sorbent molecule. Furthermore, the average interaction energy is calculated by integration of this energy profile over the entire pore characteristics length (pore width for slit-pores or radius in case of curved geometries). This implies that in a filled pore there is a continuous distribution of molecules along the characteristic length. However, micropores have dimensions comparable to molecular widths, and hence, the molecules in a filled pore can occupy only discrete positions relative to each other, if minor thermal vibrations are to be neglected. The original $\mathrm{HK}$ model has also been criticized for not taking the distribution of energy into account while calculating the average interaction energy (W ebb and Orr, 1997). A s a result of these shortcomings and others, it has been observed that pore sizes estimated by the $\mathrm{HK}$ method for materials in the higher micropore size range (8-20 $)$ ) to be unrealistically low in some cases (Carrott et al., 1998).

The above listed shortcomings in the existing model provides the motivation for developing a new model based on an improved energy profile for a micropore filled with adsorbate molecules. The new model proposed in this work considers molecules to occupy discrete positions in the adsorbate-filled micropore, and the interaction energy of a molecule is calculated using an intermolecular spacing corresponding to a minimum energy potential between that molecule and the immediate neighboring molecules. The average interaction energy is obtained by a population-weighted average of energy potentials rather than by integration. New models for PSD, based on the Horváth-Kawazoe principle, have been proposed for slit, cylindrical, as well as spherical, shaped micropores. Comparison of the average interaction energy vs. pore-size profiles for the original and modified models showed a good agreement for small pore widths, but a considerable deviation was observed at pore widths measuring more than two adsorbate molecule diameters. PSD using the modified energy profile have been obtained using isotherm data in literature for all three pore geometries, and the results are compared with that obtained using the original model.

\section{Review of the Original-HK Slit-shaped Pore Model}

The basis for obtaining the energy profile in a pore is the L ennard-J ones 6-12 potential

$$
\epsilon_{12}(z)=4 \epsilon_{12}^{*}\left[\left(\frac{\sigma}{z}\right)^{12}-\left(\frac{\sigma}{z}\right)^{6}\right]
$$

$\mathrm{H}$ alsey and coworkers gave the interaction energy of one adsorbate molecule with a single infinite-layer plane of adsor- 
bent molecules to be as follows (Sams et al., 1960)

$$
\epsilon(\mathrm{z})=\frac{\mathrm{N}_{\mathrm{s}} \mathrm{A}_{\mathrm{s}}}{2 \sigma^{4}}\left[-\left(\frac{\sigma}{\mathrm{z}}\right)^{4}+\left(\frac{\sigma}{\mathrm{z}}\right)^{10}\right]
$$

Everett and Powl (1976) extended the above result to two parallel infinite lattice planes whose nuclei are spaced at distance $\mathrm{L}$ apart

$$
\epsilon(\mathrm{z})=\frac{\mathrm{N}_{\mathrm{s}} \mathrm{A}_{\mathrm{s}}}{2 \sigma^{4}}\left[-\left(\frac{\sigma}{\mathrm{z}}\right)^{4}+\left(\frac{\sigma}{\mathrm{z}}\right)^{10}-\left(\frac{\sigma}{\mathrm{L}-\mathrm{z}}\right)^{4}+\left(\frac{\sigma}{\mathrm{L}-\mathrm{z}}\right)^{10}\right]
$$

where the internuclear distance at zero-interaction energy $(\AA): \sigma=(2 / 5)^{1 / 6} d_{0}, d_{0}$ being the average of the adsorbate and adsorbent molecule diameters $\left(\left(d_{0}+d_{A}\right) / 2\right) . N_{s}$ is the number of sorbent molecules per unit area, and $z$ is the internuclear distance between the adsorbate and adsorbent molecules $(A)$. The dispersion constants $A_{S}$ and $A_{A}$ are calculated by the Kirkwood-Muller formulae $\left(\mathrm{erg} \cdot \mathrm{cm}^{6}\right.$ ) as follows

$$
\begin{aligned}
& \mathrm{A}_{\mathrm{S}}=\frac{6 \mathrm{mc}^{2} \alpha_{\mathrm{s}} \alpha_{\mathrm{A}}}{\frac{\alpha_{\mathrm{s}}}{\chi_{\mathrm{S}}}+\frac{\alpha_{\mathrm{A}}}{\chi_{\mathrm{A}}}} \\
& \mathrm{A}_{\mathrm{A}}=\frac{3}{2} \mathrm{mc}^{2} \alpha_{\mathrm{s}} \alpha_{\mathrm{A}}
\end{aligned}
$$

Further, Horváth and Kawazoe (1983) proposed that the potential is increased by the interaction of adsorbate molecules within the pore. They included this additional interaction by adding an adsorbate dispersion term $\left(\mathrm{N}_{\mathrm{A}} \mathrm{A}_{\mathrm{A}}\right)$ term in the numerator of the depth of the potential energy minimum $\left(\mathrm{N}_{\mathrm{s}} / 2 \sigma^{4}\right)$ in $\mathrm{Eq} .3$ as follows

$$
\begin{aligned}
\epsilon(\mathrm{z})=\frac{\mathrm{N}_{\mathrm{S}} \mathrm{A}_{\mathrm{S}}+\mathrm{N}_{\mathrm{A}} \mathrm{A}_{\mathrm{A}}}{2 \sigma^{4}}\left[-\left(\frac{\sigma}{\mathrm{z}}\right)^{4}+\left(\frac{\sigma}{\mathrm{z}}\right)^{10}\right. & -\left(\frac{\sigma}{\mathrm{L}-\mathrm{z}}\right)^{4} \\
& \left.+\left(\frac{\sigma}{\mathrm{L}-\mathrm{z}}\right)^{10}\right]
\end{aligned}
$$

However, no clear justification could be found in literature for incorporating the adsorbate-adsorbate-adsorbent interaction in this manner. Further, $\epsilon(z)$ in $E q .4$ may be split as follows

$$
\begin{aligned}
\epsilon(\mathrm{z}) & =\epsilon_{\mathrm{A}-\mathrm{S}}(\mathrm{z})+\epsilon_{\mathrm{A}-\mathrm{A}}(\mathrm{z}) \\
& =\frac{\mathrm{N}_{\mathrm{S}} \mathrm{A}_{\mathrm{S}}}{2 \sigma^{4}}\left[-\left(\frac{\sigma}{\mathrm{z}}\right)^{4}+\left(\frac{\sigma}{\mathrm{z}}\right)^{10}-\left(\frac{\sigma}{\mathrm{L}-\mathrm{z}}\right)^{4}+\left(\frac{\sigma}{\mathrm{L}-\mathrm{z}}\right)^{10}\right] \\
+ & \frac{\mathrm{N}_{\mathrm{A}} \mathrm{A}_{\mathrm{A}}}{2 \sigma^{4}}\left[-\left(\frac{\sigma}{\mathrm{z}}\right)^{4}+\left(\frac{\sigma}{\mathrm{z}}\right)^{10}-\left(\frac{\sigma}{\mathrm{L}-\mathrm{z}}\right)^{4}+\left(\frac{\sigma}{\mathrm{L}-\mathrm{z}}\right)^{10}\right]
\end{aligned}
$$

The first term $\epsilon_{\mathrm{A}-\mathrm{s}}(\mathrm{z})$ gives the adsorbate-surface interaction and the second term $\epsilon_{\mathrm{A}-\mathrm{A}}(\mathrm{z})$ gives the adsorbate-adsorbate interaction. Since the distance $z$ of the gas molecule is the same in both $\epsilon_{A-S}(z)$, as well as $\epsilon_{A-A}(z), E q .6$ gives an erroneous interpretation that the adsorbate-adsorbate interaction is caused between a gas molecule and two parallel infinite sheets of gas molecules, impractically placed at the same position as the sorbent molecules. Besides, the internuclear distance at zero interaction energy $(\sigma)$ to be used in $\epsilon_{\mathrm{A}-\mathrm{A}}(\mathrm{z})$ is expected to be different from that used in the $\epsilon_{\mathrm{A}-\mathrm{S}}(\mathrm{z})$ expression, but the original model does not take this fact into account.

The next step in the derivation was obtaining the average interaction energy by integrating the above profile over the free space in the slit-pore

$$
\bar{\epsilon}(z)=\frac{\int_{d_{0}}^{L-d_{0}} \epsilon(z) d z}{\int_{d_{0}}^{L-d_{0}} d z}
$$

This implies that a gas molecule has a continuous distribution of positions in the filled micropore, which it is free to occupy. In reality, however, the gas molecule has only fixed positions at which it can exist when the pore is filled to capacity, especially when the pore width is only a few multiples of the adsorbate molecule diameter. Besides, the Boltzmann law of distribution of energy requires that the above average be instead computed by weighing the energy terms with the probability of a molecule to possess that energy, namely $\mathrm{e}^{-\epsilon / \mathrm{KT}}$. In other words, the molecule is expected to occupy a position at which the energy potential is minimum. This aspect was not considered in the original work. In the present work, a discretized average is proposed which attempts to incorporate the above features in the model.

Finally, the average potential energy so calculated was related to the free energy change upon adsorption $R T \operatorname{In}\left(P / P_{0}\right)$. The resulting slit-pore $\mathrm{HK}$ model is as follows

$$
\begin{aligned}
\mathrm{RT} \operatorname{In}\left(\frac{\mathrm{P}}{\mathrm{P}_{\mathrm{o}}}\right) & =\mathrm{N}_{\mathrm{A} \nu} \cdot \bar{\epsilon}(\mathrm{z})=\mathrm{N}_{\mathrm{A} \nu} \frac{\mathrm{N}_{\mathrm{s}} \mathrm{A}_{\mathrm{S}}+\mathrm{N}_{\mathrm{A}} \mathrm{A}_{\mathrm{A}}}{\sigma^{4}\left(\mathrm{~L}-2 \mathrm{~d}_{\mathrm{o}}\right)} \\
\times & {\left[\frac{\sigma^{4}}{3\left(\mathrm{~L}-\mathrm{d}_{\mathrm{o}}\right)^{3}}-\frac{\sigma^{10}}{9\left(\mathrm{~L}-\mathrm{d}_{\mathrm{o}}\right)^{9}}-\frac{\sigma^{4}}{3 \mathrm{~d}_{0}^{3}}+\frac{\sigma^{10}}{9 \mathrm{~d}_{\mathrm{o}}^{9}}\right] }
\end{aligned}
$$

As can be seen from Eq. 8, the interaction energy of a molecule is indeterminate when $L=2 d_{0}$, that is, then the free space of the pore $\left(L-d_{s}\right)$ equals the diameter of an adsorbate molecule $\left(d_{A}\right)$. However, a finite value for the potential exists for a molecule of this size as is obvious from Eq. 5 . This anomaly is overcome in the modified model.

An implicit assumption in the original derivation by H orváth and Kawazoe (1983) was that the isotherm followed Henry's law. It is well known that most isotherm data show a considerable deviation from Henry's law at pressures near the saturation pressure of the adsorbate, and the equilibrium loading usually follows a Langmuir-type behavior. Cheng and 
$Y$ ang (1994) introduced a correction term to incorporate the Langmuir isotherm in the model and the corrected model (referred henceforth as the $\mathrm{HK}-\mathrm{CY}$ model) was given as

$$
\begin{aligned}
R T \ln \left(\frac{\mathrm{P}}{\mathrm{P}_{\mathrm{o}}}\right) & +\mathrm{RT}\left[1+\frac{1}{\theta} \ln (1-\theta)\right] \\
& =\mathrm{N}_{\mathrm{A} \nu} \cdot \bar{\epsilon}(\mathrm{z})=\mathrm{N}_{\mathrm{A} \nu} \frac{\mathrm{N}_{\mathrm{S}} \mathrm{A}_{\mathrm{S}}+\mathrm{N}_{\mathrm{A}} \mathrm{A}_{\mathrm{A}}}{\sigma^{4}\left(\mathrm{~L}-2 \mathrm{~d}_{\mathrm{o}}\right)} \\
& \times\left[\frac{\sigma^{4}}{3\left(\mathrm{~L}-\mathrm{d}_{\mathrm{o}}\right)^{3}}-\frac{\sigma^{10}}{9\left(\mathrm{~L}-\mathrm{d}_{\mathrm{o}}\right)^{9}}-\frac{\sigma^{4}}{3 \mathrm{~d}_{0}^{3}}+\frac{\sigma^{10}}{9 \mathrm{~d}_{\mathrm{o}}^{9}}\right]
\end{aligned}
$$

The Langmuir isotherm is typically represented as follows

$$
\theta=\frac{K\left(\frac{\mathrm{P}}{\mathrm{P}_{\mathrm{o}}}\right)}{1+K\left(\frac{\mathrm{P}}{\mathrm{P}_{\mathrm{o}}}\right)}
$$

Taking a limit of $\mathrm{E} \mathrm{q.} 10$ as $\mathrm{P}$ approaches the saturation pressure $\mathrm{P}_{0}$, we have $\theta=\mathrm{K} /(1+\mathrm{K})$. Now, applying the above limit of $P / P_{0}$ tending to 1 to $E q .9$, we obtain the following

$$
\lim _{P \rightarrow P_{0}} N_{A \nu} \cdot \bar{\epsilon}(z)=R T\left[1-\frac{K+1}{K} \ln (K+1)\right]
$$

Thus, at high pressures, the average interaction energy should tend to the value given by $\mathrm{Eq}$. 11. Near the saturation pressure, the size of the largest pore, which can be filled with adsorbate, tends towards infinity. As can be seen from Eq. 8, as $L \rightarrow \infty$, the average interaction energy tends towards zero. Also, intuitively, is apparent that there will always be some adsorbate-adsorbate interaction and the limiting interaction energy cannot go to zero even at large pore size. Thus, the original model for average interaction energy in the pore is inconsistent with the $\mathrm{HK}$ equation with the $\mathrm{C}$ heng- $\mathrm{Y}$ ang correction. The proposed model is found to have a better agreement in this regard. The physical parameters used in the micropore size distribution calculations in this work are given in Table 1.

\section{Modified HK Model for Slit-shaped Pores}

The general strategy for obtaining the PSD for the three pore geometries, namely, slit, cylindrical, and spherical pores is basically the same. The modified model for calculating the average interaction energy in a filled micropore proceeds by first estimating the number of gas adsorbate molecule layers within the filled micropore. Each gas molecule is assumed to rest preferentially at a position at which its energy potential within the pore would be minimum in accordance with the Boltzmann law of energy distribution. Furthermore, a gas molecule is assumed to interact most effectively with only its laterally immediate molecular layer. This fact is in agreement with experimental observations which show that the energy of adsorption of a monolayer of adsorbate molecules on a clean sorbent surface is the highest and that on subsequently adsorbed layers of gas molecules is a nearly constant lower value. A ny interaction with molecules not in the immediate proximity of the molecule or with those lying above or below the molecule in the same layer is assumed to be negligible. The average interaction energy is then calculated by averaging the energy potentials of the individual gas molecule layers weighted by an approximate molecular population of each layer.

Consider a slit pore with the nuclei of the sorbent molecules in the lattice planes forming the pore-wall spaced at a dis-

\begin{tabular}{|c|c|c|c|c|c|}
\hline \multirow[b]{2}{*}{ Parameter } & \multicolumn{3}{|c|}{ A dsorbent } & \multicolumn{2}{|c|}{ A dsorbate } \\
\hline & Carbon & $\begin{array}{l}\text { A luminosilicate } \\
\text { Oxide Ion }\end{array}$ & $\begin{array}{c}\text { Aluminophosphate } \\
\text { Oxide Ion }\end{array}$ & N itrogen & Argon \\
\hline Dia. $(\AA)$ & $3.40 *$ & $2.76 * *$ & $2.60 * *$ & $\begin{array}{l}3.00 * \\
372\|\|, \|\end{array}$ & $\begin{array}{c}3.82^{\dagger \dagger, \ddagger, \ddagger \ddagger, \| ~} \\
295^{\dagger \S \S}\end{array}$ \\
\hline Polarizability, $\alpha\left(\mathrm{cm}^{3}\right)$ & $1.02 \times 10^{-24^{*}}$ & $2.5 \times 10^{-24 * *}$ & $2.5 \times 10^{-24^{* *}}$ & $1.46 \times 10^{-24 *}$ & $1.63 \times 10^{-24 * *}$ \\
\hline $\begin{array}{c}\text { M agnetic } \\
\text { Susceptibility } \times\left(\mathrm{cm}^{3}\right)\end{array}$ & $1.35 \times 10^{-28^{*}}$ & $1.3 \times 10^{-29 * *}$ & $1.3 \times 10^{-29 * *}$ & $2.0 \times 10^{-29 *}$ & $3.24 \times 10^{-29 * *}$ \\
\hline $\begin{array}{l}\text { M olec. } \\
\text { Surface density } \\
\left(\text { M olecule } / \mathrm{cm}^{2}\right)\end{array}$ & $3.85 \times 10^{15^{*}}$ & $\begin{array}{c}3.75 \times 10^{\dagger, \S \S} \\
1.00 \times 10^{15 \dagger \dagger, \S} \\
8.48 \times 10^{14 \ddagger}, * * \\
8.73 \times 10^{14 \neq \ddagger}, * * \\
3.27 \times 10^{14\|\|, \S}\end{array}$ & $1.0 \times 10^{15 \S}$ & $6.7 \times 10^{14^{*}}$ & $8.52 \times 10^{14^{* *}}$ \\
\hline
\end{tabular}
tance $L$ apart. If the diameter of the sorbent molecules is denoted as $d_{S}$ and that of the adsorbent molecules as $d_{A}$, then the number of molecule layers $M$ that can be accommo-

Table 1. Physical Parameters Used in M icropore Size Distribution Calculations

*H orváth and Kawazoe (1983).

** Cheng and $Y$ ang (1994).

+ F aujasite, Slit-pore model.

${ }^{\dagger+}$ ZSM -5, Cylindrical-pore model.

$\neq$ F aujasite, Spherical-pore model.

$\neq \ddagger$ A Zeolite, Spherical-pore model

$\S$ Estimated in this work.

§§ A SA P $2010 \mathrm{M}$ anual, M icromeritics Inc. (1995).

"R azmus and $\mathrm{H}$ all (1991).

IIIIM CM -41, Cylindrical-pore model. 


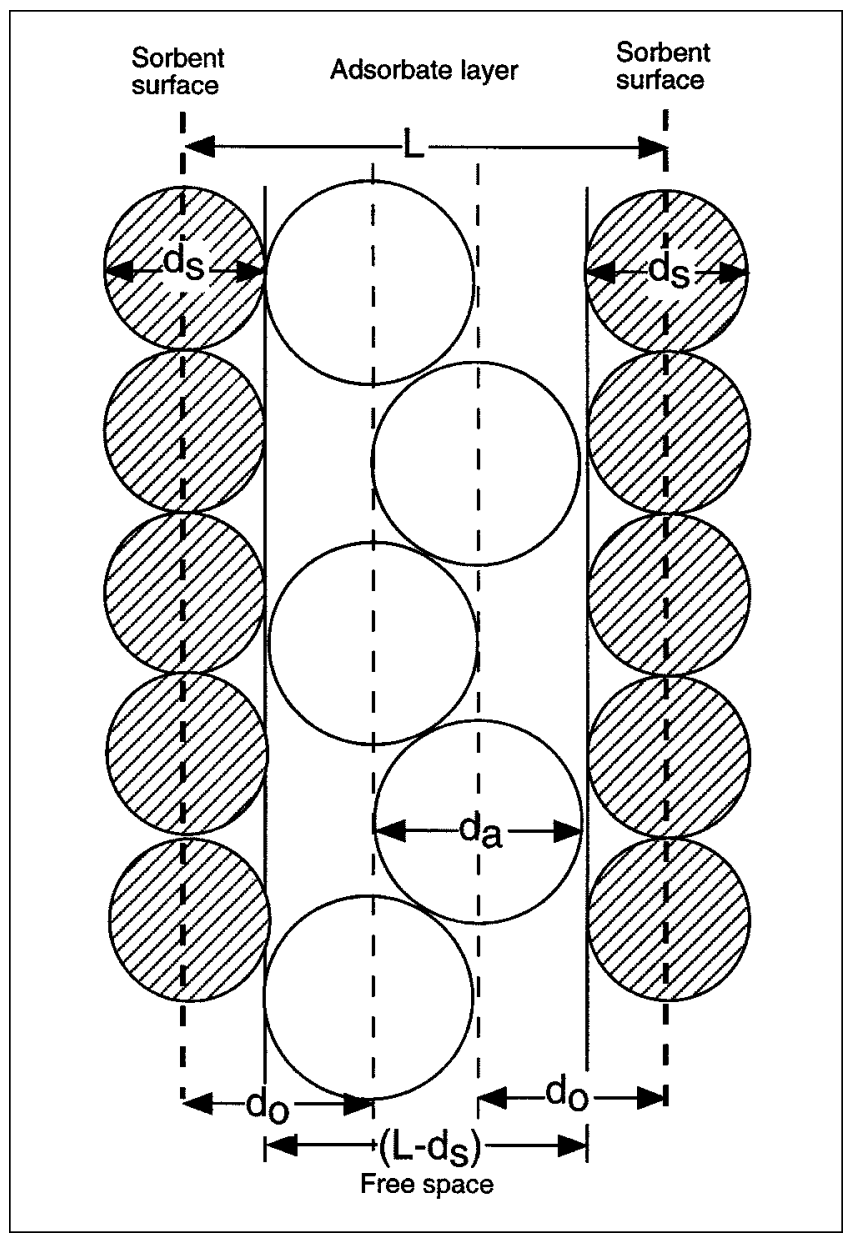

Figure 1. Single layer of adsorbate molecules in a slit-shaped adsorbent pore of width $L$.

dated laterally as the pore gets filled up can be estimated as

$$
M=\frac{L-d_{S}}{d_{A}}
$$

It should be noted that $M$ can be a whole number or it may also be a fraction. The physical interpretation of a fractional number of layers is that the molecules in the layers are not arranged with their centers oriented in the same straight line, but are slightly skewed with respect to each other so as to afford a greater packing of molecules. Such a packing arrangement will, of course, result in a decrease in molecular density in the neighboring layers, but the resulting decrease in interaction energy will be accounted for when taking the average of the energy potentials.

When $1 \leq \mathrm{M}<2$, only one layer of molecules can be accommodated within the slit pore, as shown in Figure 1. In this case, each adsorbate molecule will interact with the two lattice planes forming the pore wall of the sorbent. The interaction energy of this monolayer, denoted as $\epsilon_{1}(z)$, is given by an expression similar to $\mathrm{Eq}$. 3. A n examination of $\mathrm{Eq}$. 3 reveals that the minimum energy potential exists at a distance of $d_{0}$ from either sorbent lattice planes. Since the B oltzmann law of energy distribution law suggests that the molecule will most probably rest at a position at which the energy potential is the minimum, each molecule is assumed to exist at a distance of $d_{0}$ from one of the two sorbent lattice planes. The interaction energy would then be

$$
\begin{aligned}
\epsilon_{1}(z)=\frac{\mathrm{N}_{\mathrm{s}} \mathrm{A}_{\mathrm{s}}}{2 \sigma_{\mathrm{s}}^{4}}\left[-\left(\frac{\sigma^{\mathrm{s}}}{\mathrm{d}_{\mathrm{o}}}\right)^{4}+\left(\frac{\sigma_{\mathrm{s}}}{\mathrm{d}_{\mathrm{o}}}\right)^{10}-\left(\frac{\sigma_{\mathrm{s}}}{\mathrm{L}-\mathrm{d}_{\mathrm{o}}}\right)^{4}\right. \\
\left.+\left(\frac{\sigma_{\mathrm{s}}}{\mathrm{L}-\mathrm{d}_{\mathrm{o}}}\right)^{10}\right]
\end{aligned}
$$

N ote that the internuclear distance at zero interaction energy for an adsorbate-sorbent system is now denoted as $\sigma_{\mathrm{s}}$ to differentiate it from $\sigma_{\mathrm{A}}$, which is the zero interaction energy distance for an adsorbate-adsorbate system. The expressions for $\sigma_{\mathrm{S}}$ and $\sigma_{\mathrm{A}}$ are as follows

$$
\begin{aligned}
& \sigma_{\mathrm{S}}=(2 / 5)^{1 / 6} \mathrm{~d}_{\mathrm{o}} \\
& \sigma_{\mathrm{A}}=(2 / 5)^{1 / 6} \mathrm{~d}_{\mathrm{A}}
\end{aligned}
$$

The average energy of a slit pore having $1<M<2$ is the same as $\epsilon_{\mathrm{l}}(z)$ since, irrespective of $M$, the molecules are assumed to rest at a distance if $d_{0}$ from either pore walls. The situation is slightly different when $M \geq 2$. Three cases for this situation are depicted in Figures 2-4, wherein two different types of layers can be visualized: the first type consisting of the two layers in the immediate vicinity of the two pore walls, and at a distance of $d_{0}$ from them, and the second type of molecules present in the interior of the pore sandwiched between two adjoining adsorbate layers. The first type of molecules (marked A in the figure) will interact with the sorbent molecules on one side and with adsorbate molecules on the other. The distance between the two adsorbate molecules would be approximately $d_{A}$ by the same argument as discussed above. The interaction energy of this layer of molecules, denoted as $\epsilon_{2}(z)$ is given by

$$
\begin{aligned}
\epsilon_{2}(\mathrm{z})=\frac{\mathrm{N}_{\mathrm{s}} \mathrm{A}_{\mathrm{s}}}{2 \sigma_{\mathrm{s}}{ }^{4}}\left[-\left(\frac{\sigma_{\mathrm{s}}}{\mathrm{d}_{\mathrm{o}}}\right)^{4}\right. & \left.+\left(\frac{\sigma_{\mathrm{s}}}{\mathrm{d}_{\mathrm{o}}}\right)^{10}\right] \\
& +\frac{\mathrm{N}_{\mathrm{A}} \mathrm{A}_{\mathrm{A}}}{2 \sigma_{\mathrm{A}}^{4}}\left[-\left(\frac{\sigma_{\mathrm{A}}}{\mathrm{d}_{\mathrm{A}}}\right)^{4}+\left(\frac{\sigma_{\mathrm{A}}}{\mathrm{d}_{\mathrm{A}}}\right)^{10}\right]
\end{aligned}
$$

The second type of molecules (marked B in the figure), comprising $(n-2)$ number of layers, will interact effectively only with two parallel planes of gas molecules at a distance of $d_{A}$ on either side of the molecule and its interaction energy $\epsilon_{3}(z)$ will be given by

$$
\epsilon_{3}(\mathrm{z})=2 \cdot \frac{\mathrm{N}_{\mathrm{A}} \mathrm{A}_{\mathrm{A}}}{2 \sigma_{\mathrm{A}}^{4}}\left[-\left(\frac{\sigma_{\mathrm{A}}}{\mathrm{d}_{\mathrm{A}}}\right)^{4}+\left(\frac{\sigma_{\mathrm{A}}}{\mathrm{d}_{\mathrm{A}}}\right)^{10}\right]
$$

The average interaction energy for $M>2$ can be calculated by weighing the energy potentials of the molecules by 


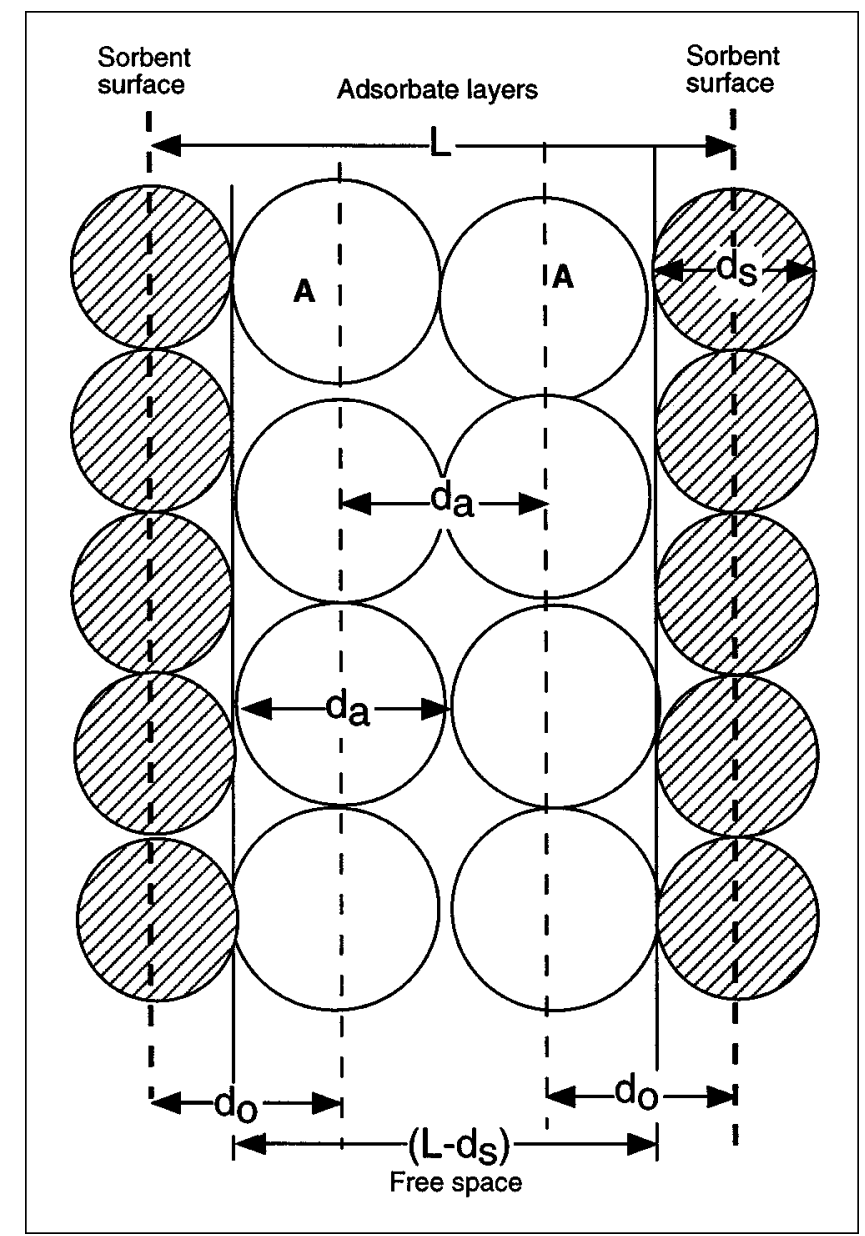

Figure 2. Two layers of adsorbate molecules in a slit-shaped adsorbent pore of width $L$.

the actual number of layers of molecules possessing the corresponding energy

$$
\bar{\epsilon}=\frac{2 \epsilon_{2}+(M-2) \epsilon_{3}}{M}
$$

Here, $M$, the number of layers accommodated within the pore, is given by $\mathrm{Eq}$. 12. The above method of averaging does away with integration, which assumes that a gas molecule in a filled micropore is free to assume a continuous distribution of positions within the slit pore free space. When $M$ is not a whole number, the picture will be something between the two whole numbers enclosing $M$. For example, Figure 2 shows the molecule layers for $M=2$ and Figure 3 shows the case when $\mathrm{M}=3$. For $2<\mathrm{M}<3$, Figure 4 shows a likely configuration. Since the centers of the sorbate molecules will no longer be collinear, the density of the molecules adjacent to the middle layer will be decreased and its interaction energy will be lower. This factor is taken into account by using the interpolating Eq. 18, which will consider only a fraction of the adsorbate-adsorbate potential $\epsilon_{3}(\mathrm{z})$ for this molecule in the final average. Equation 18 will thus be exact when $M$ is a whole number, and will be an interpolated approximation when $M$ lies between two whole numbers.

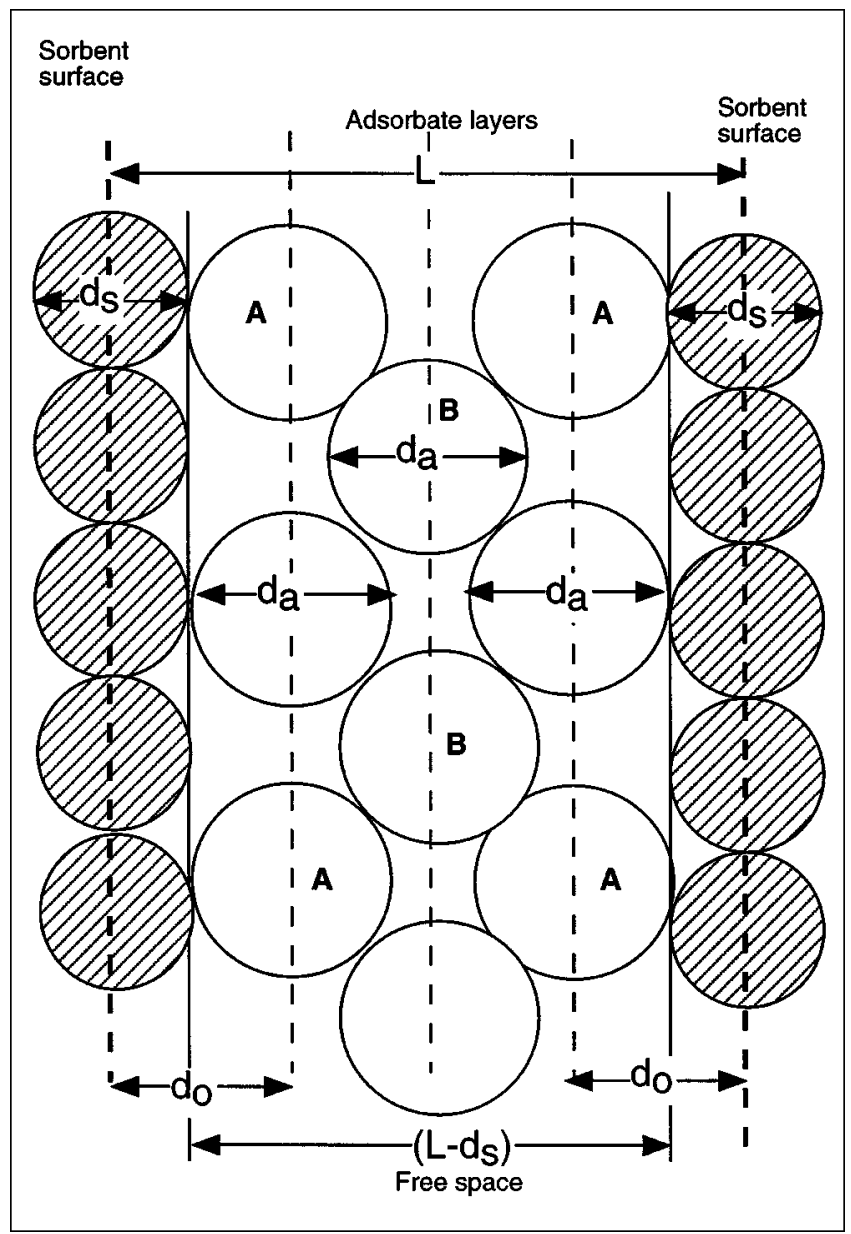

Figure 3. Three layers of adsorbate molecules in a slit-shaped adsorbent pore of width $L$.

The pore-size distribution can now be determined following the methodology outlined by Horváth and Kawazoe (1983). As was explained in the previous section regarding the original $\mathrm{HK}$ model, the free energy change upon adsorption, $\mathrm{RT} \ln \left(\mathrm{P} / \mathrm{P}_{\mathrm{o}}\right)$, is equated to the molar average interaction energy $\mathrm{N}_{\mathrm{A} \nu} \cdot \bar{\epsilon}$. Thus, the modified-HK equation can be written as

$$
\mathrm{RT} \ln \left(\frac{\mathrm{P}}{\mathrm{P}_{\mathrm{o}}}\right)=\mathrm{N}_{\mathrm{A} \nu} \cdot \bar{\epsilon}
$$

On applying the correction for isotherm nonlinearity given by Cheng and $Y$ ang (1994), the modified HK-CY equation is obtained as

$$
\mathrm{RT} \ln \left(\frac{\mathrm{P}}{\mathrm{P}_{\mathrm{o}}}\right)+\mathrm{RT}\left[1+\frac{1}{\theta} \ln (1-\theta)\right]=\mathrm{N}_{\mathrm{A} \nu} \cdot \bar{\epsilon}
$$

In E qs. 19 and 20 above

$$
\begin{aligned}
& \bar{\epsilon}=\epsilon_{1} \\
& \text { when } 1 \leq M<2 \\
& =\frac{2 \epsilon_{2}+(M-2) \epsilon_{3}}{M} \text { when } M \geq 2
\end{aligned}
$$




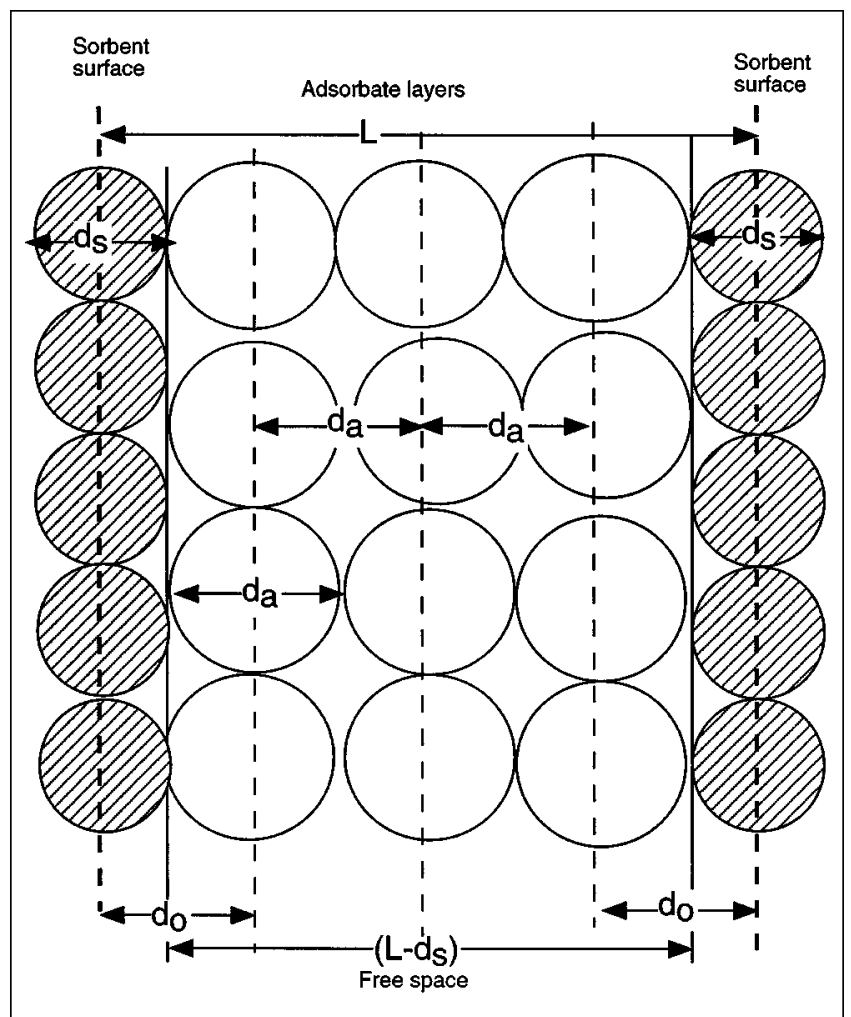

Figure 4. Possible configuration of adsorbate molecules in a slit-shaped adsorbent pore measuring between 2 to 3 adsorbate molecule diameters in width.

The above model is simple and can be easily implemented using a programming code or a spreadsheet. A slight discontinuity in $\bar{\epsilon}$ may occur at $M=2$ as a result of the above two interpolating functions, but the difference in the values was found to be negligibly small for the examples studied in this work. The algorithm for determining pore size using the corrected $\mathrm{HK}$ equation is summarized in Table 2.

\section{Review or Original HK Model for Cylindrical Pores}

The interaction energy of a gas molecule in a cylindrical pore composed of a single layer of oxide ions was first obtained by E verett and Powl (1976) and is given as follows

$$
\begin{aligned}
\epsilon(\mathrm{r})=\frac{5}{2} \pi \epsilon^{*}\left[\frac{21}{32}\left(\frac{\mathrm{d}_{\mathrm{o}}}{\mathrm{L}}\right)^{10} \sum_{\mathrm{k}=0}^{\infty} \alpha_{\mathrm{k}}\left(\frac{\mathrm{r}}{\mathrm{L}}\right)^{2 \mathrm{k}}\right. \\
\\
\left.\quad-\left(\frac{\mathrm{d}_{\mathrm{o}}}{\mathrm{L}}\right)^{4} \sum_{\mathrm{k}=0}^{\infty} \beta_{\mathrm{k}}\left(\frac{\mathrm{r}}{\mathrm{L}}\right)^{2 \mathrm{k}}\right]
\end{aligned}
$$

In the above equation, the constants $\alpha_{\mathrm{k}}$ and $\beta_{\mathrm{k}}$ are given by

$$
\alpha_{\mathrm{k}}=\left(\frac{-4.5-\mathrm{k}}{\mathrm{k}}\right)^{2} \alpha_{\mathrm{k}-1}
$$

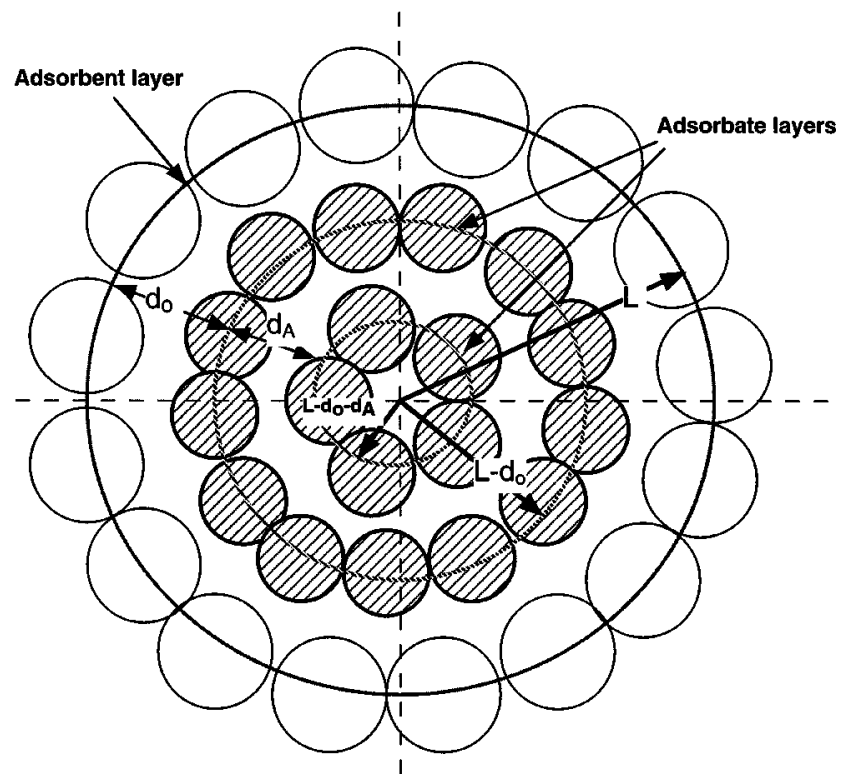

Figure 5. Cross-sectional view of the configuration of adsorbate molecules in a cylindrical pore of radius $L$.

$$
\beta_{\mathrm{k}}=\left(\frac{-1.5-\mathrm{k}}{\mathrm{k}}\right)^{2} \beta_{\mathrm{k}-1}
$$

where $\alpha_{0}=\beta_{0}=1$. Here, $r$ is the distance of the nucleus of gas molecule from the central axis of the cylinder and $\epsilon^{*}$ is the potential energy minimum given by

$$
\epsilon^{*}=\frac{3}{10} \frac{\mathrm{N}_{\mathrm{S}} \mathrm{A}_{\mathrm{S}}}{\mathrm{d}_{\mathrm{o}}^{4}}
$$

U sing the result, Saito and Foley (1991) developed a PSD model using a method similar to that of Horváth and Kawazoe (1983). In their work also, the adsorbate-adsorbate-adsorbent interaction was incorporated in the model by modifying the potential energy minimum $\epsilon^{*}$ to the following

$$
\epsilon^{*}=\frac{3}{10} \frac{\mathrm{N}_{\mathrm{S}} \mathrm{A}_{\mathrm{S}}+\mathrm{N}_{\mathrm{A}} \mathrm{A}_{\mathrm{A}}}{\mathrm{d}_{0}^{4}}
$$

The resulting radial energy potential profile was then averaged over the cylindrical cross-sectional area to give the following original-HK equation for cylindrical pores

$$
\begin{array}{r}
\ln \left(\frac{\mathrm{P}}{\mathrm{P}_{\mathrm{o}}}\right)=\frac{3}{4} \pi \mathrm{N}_{\mathrm{A} \nu} \frac{\mathrm{N}_{\mathrm{s}} \mathrm{A}_{\mathrm{S}}+\mathrm{N}_{\mathrm{A}} \mathrm{A}_{\mathrm{A}}}{\mathrm{d}_{\mathrm{o}}^{4}} \sum_{\mathrm{k}=0}^{\infty}\left\{\frac{1}{\mathrm{k}+1}\left(1-\frac{\mathrm{d}_{\mathrm{o}}}{\mathrm{L}}\right)^{2 \mathrm{k}}\right. \\
\left.\times\left[\frac{21}{32} \alpha_{\mathrm{k}}\left(\frac{\mathrm{d}_{\mathrm{o}}}{\mathrm{L}}\right)^{10}-\beta_{\mathrm{k}}\left(\frac{\mathrm{d}_{\mathrm{o}}}{\mathrm{L}}\right)^{4}\right]\right\}
\end{array}
$$


Table 2. Algorithm (Pseudo-C ode) for Obtaining Effective Pore Size of Slit-Shaped Pores

(1) Guess slit width $L$ corresponding to adsorbate relative pressure $P / P_{0}$.

(2) Calculate number of adsorbate molecular layers M (E q. 12) $M=\frac{L-d_{s}}{d_{A}}$

(3) Calculate $\epsilon_{1}, \epsilon_{2}$, and $\epsilon_{3}$ (E qs. 13, 16, and 17). $\epsilon_{1}(z)=\frac{N_{s} A_{s}}{2 \sigma_{s}^{4}}\left[-\left(\frac{\sigma_{s}}{d_{0}}\right)^{4}+\left(\frac{\sigma_{s}}{d_{0}}\right)^{10}\right.$

$$
\left.-\left(\frac{\sigma_{\mathrm{s}}}{\mathrm{L}-\mathrm{d}_{0}}\right)^{4}+\left(\frac{\sigma_{\mathrm{s}}}{\mathrm{L}-\mathrm{d}_{0}}\right)^{10}\right]
$$

$\epsilon_{2}(z)=\frac{\mathrm{N}_{\mathrm{s}} \mathrm{A}_{\mathrm{s}}}{2 \sigma_{\mathrm{s}}^{4}}\left[-\left(\frac{\sigma_{\mathrm{s}}}{\mathrm{d}_{0}}\right)^{4}+\left(\frac{\sigma_{\mathrm{s}}}{\mathrm{d}_{0}}\right)^{10}\right]$

$$
+\frac{\mathrm{N}_{\mathrm{A}} \mathrm{A}_{\mathrm{A}}}{2 \sigma_{\mathrm{A}}^{4}}\left[-\left(\frac{\sigma_{\mathrm{A}}}{\mathrm{d}_{\mathrm{A}}}\right)^{4}+\left(\frac{\sigma_{\mathrm{A}}}{\mathrm{d}_{\mathrm{A}}}\right)^{10}\right]
$$

$\epsilon_{3}(\mathrm{z})=2 \cdot \frac{\mathrm{N}_{\mathrm{A}} \mathrm{A}_{\mathrm{A}}}{2 \sigma_{\mathrm{A}}^{4}}\left[-\left(\frac{\sigma_{\mathrm{A}}}{\mathrm{d}_{\mathrm{A}}}\right)^{4}+\left(\frac{\sigma_{\mathrm{A}}}{\mathrm{d}_{\mathrm{A}}}\right)^{10}\right]$

(4) If $M<2$ then

$$
\bar{\epsilon}=\epsilon_{1}
$$

Else $(M>2)$

$$
\bar{\epsilon}=\frac{2 \epsilon_{2}+(M-2) \epsilon_{3}}{M}
$$

(5) If $\left[R T \ln \left(P / P_{0}\right)=N_{A V} \bar{\epsilon}\right]$ then

Effective pore width $=\left(\mathrm{L}-\mathrm{d}_{\mathrm{S}}\right)$

Else

Guess new $L$ and iterate from (1) again.

On applying the Cheng-Y ang correction discussed above, the original $\mathrm{HK}-\mathrm{CY}$ equation is obtained as follows

$$
\begin{aligned}
& \ln \left(\frac{\mathrm{P}}{\mathrm{P}_{\mathrm{o}}}\right)+\mathrm{RT}\left[1+\frac{1}{\theta} \ln (1-\theta)\right]=\frac{3}{4} \pi \mathrm{N}_{\mathrm{A} \nu} \frac{\mathrm{N}_{\mathrm{s}} \mathrm{A}_{\mathrm{s}}+\mathrm{N}_{\mathrm{A}} \mathrm{A}_{\mathrm{A}}}{\mathrm{d}_{\mathrm{o}}^{4}} \\
& \quad \times \sum_{\mathrm{k}=0}^{\infty}\left[\frac{1}{\mathrm{k}+1}\left(1-\frac{\mathrm{d}_{\mathrm{o}}}{\mathrm{L}}\right)^{2 \mathrm{k}}\left(\frac{21}{32} \alpha_{\mathrm{k}}\left(\frac{\mathrm{d}_{\mathrm{o}}}{\mathrm{L}}\right)^{10}-\beta_{\mathrm{k}}\left(\frac{\mathrm{d}_{\mathrm{o}}}{\mathrm{L}}\right)^{4}\right)\right]
\end{aligned}
$$

This model also suffers from the same drawbacks as those discussed for the slit-pore model, namely, an erroneous method to calculate the adsorbate-adsorbate interaction which underestimates the energy potential for large pore diameters.

\section{Modified HK Model for Cylindrical Pores}

The derivation for the modified HK model for pores with cylindrical geometry follows the same procedure as that for the slit-pore model. The total number of concentric layers of adsorbate molecules that can be accomodated in the cylinder of radius $L$ is first determined. The energy potential of the adsorbate molecules in each layer is then calculated using the appropriate equation corresponding to Eq. 22. The number of sorbate molecules in each sorbate layer is estimated using the sorbate molecule diameter and diameter of the corresponding concentric layers. The average interaction energy is then determined by a population-weighted average of the individual layer potentials. O ne of the assumptions made by the model is that since adsorption will proceed from the extreme periphery of the pore towards the center, the energy potential of each layer would correspond to only the field induced by the immediately surrounding outer layer of molecules. The interaction energy induced by the inside layer of molecules enclosed by the molecules for which the energy potential is being calculated is considered to be negligible.

Figure 5 shows a cross-sectional view of a cylindrical pore. The total number of concentric molecular layers $M$ that can be accommodated within a free space diameter of $\left(2 L-d_{s}\right)$ is given by

$$
M=\operatorname{int}\left[\frac{\left(\frac{2 L-d_{s}}{d_{A}}-1\right)}{2}\right]+1
$$

Note that "int" is a mathematical function which truncates the decimal part of a value and retains only the integer part.

The first layer of molecules will be enclosed by a layer of molecules belonging to the sorbent material (such as oxide ions). Since it is assumed that molecules in a layer will preferentially occupy positions at which the energy potential is minimum, the first layer of molecules is supposed to exist at a radius of $\left(L-d_{0}\right)$ from the center. Thus, making the required substitution, the energy potential of the first layer $\left(\epsilon_{1}\right)$ is obtained as

$\epsilon_{1}=\frac{3}{4} \pi \frac{\mathrm{N}_{\mathrm{s}} \mathrm{A}_{\mathrm{s}}}{\mathrm{d}_{\mathrm{o}}^{4}}\left[\frac{21}{32} \mathrm{a}_{1}^{10} \sum_{\mathrm{k}=0}^{\infty} \alpha_{\mathrm{k}}\left(\mathrm{b}_{1}^{2 \mathrm{k}}\right)-\mathrm{a}_{1}^{4} \sum_{\mathrm{k}=0}^{\infty} \beta_{\mathrm{k}}\left(\mathrm{b}_{1}^{2 \mathrm{k}}\right)\right]$

where $a_{1}$ and $b_{1}$ are given

$$
a_{1}=\frac{d_{0}}{L} \quad b_{1}=\frac{L-d_{0}}{L}
$$

As can be inferred from Eq. 29b, the constant $a_{1}$ (and, in general, $a_{1}$ ) corresponds to a ratio of internuclear spacing $d_{0}$ to the radius of the enclosing layer of molecules, while $b_{1}$ (and, in general, $b_{i}$ ) is the ratio of distance of the layer from the central axis of the pore to the radius of the enclosing layer of molecules. Note that, in the following discussion, the layers are numbered in increasing numbers starting from the outermost layer to the innermost.

The second layer of molecules will be encircled by a cylindrical shell of adsorbate molecules. The depth of energy potential minimum will thus be different from that used in $\mathrm{Eq}$. 29a. Moreover, the internuclear spacing between the molecules is expected to be $d_{A}$ rather than $d_{0}$ and the layer of molecules is consequently located at a radius of $\left(L-d_{0}-\right.$ $d_{A}$ ) from the central axis. Thus, the energy potential of the second layer is given analogous to Eqs. $29 \mathrm{a}$ and $29 \mathrm{~b}$ and as follows

$$
\epsilon_{2}=\frac{3}{4} \pi \frac{\mathrm{N}_{\mathrm{A}} \mathrm{A}_{\mathrm{A}}}{\mathrm{d}_{\mathrm{A}}^{4}}\left[\frac{21}{32} \mathrm{a}_{\mathrm{z}}^{10} \sum_{\mathrm{k}=0}^{\infty} \mathrm{a}_{\mathrm{k}}\left(\mathrm{b}_{2}^{2 \mathrm{k}}\right)-\mathrm{a}_{2}^{4} \sum_{\mathrm{k}=0}^{\infty} \beta_{\mathrm{k}}\left(\mathrm{b}_{2}^{2 \mathrm{k}}\right)\right]
$$


where

$$
a_{2}=\frac{d_{A}}{L-d_{0}} \quad b_{2}=\frac{L-d_{0}-d_{A}}{L-d_{0}}
$$

Similarly, the third layer will exist at a distance of $\left(L-d_{0}\right.$ $-2 d_{A}$ ) from the axis enclosed by a cylindrical shell of radius $\left(L-d_{0}-d_{A}\right)$. Hence

$$
\epsilon_{3}=\frac{3}{4} \pi \frac{\mathrm{N}_{\mathrm{A}} \mathrm{A}_{\mathrm{A}}}{\mathrm{d}_{\mathrm{A}}^{4}}\left[\frac{21}{32} \mathrm{a}_{3}^{10} \sum_{\mathrm{k}=0}^{\infty} \alpha_{\mathrm{k}}\left(\mathrm{b}_{3}^{2 \mathrm{k}}\right)-\mathrm{a}_{3}^{4} \sum_{\mathrm{k}=0}^{\infty} \beta_{\mathrm{k}}\left(\mathrm{b}_{3}^{2 \mathrm{k}}\right)\right]
$$

where

$$
a_{3}=\frac{d_{A}}{L-d_{0}-d_{A}} \quad b_{3}=\frac{L-d_{0}-2 d_{A}}{L-d_{0}-d_{A}}
$$

Genearlizing the above equations, one can write down the equation for the energy potential for the $\mathrm{i}^{\text {th }}$ layer $(i>1)$ as follows

$$
\epsilon_{\mathrm{i}}=\frac{3}{4} \pi \frac{\mathrm{N}_{\mathrm{A}} \mathrm{A}_{\mathrm{A}}}{\mathrm{d}_{\mathrm{A}}^{4}}\left[\frac{21}{32} \mathrm{a}_{\mathrm{i}}^{10} \sum_{\mathrm{k}=0}^{\infty} \alpha_{\mathrm{k}}\left(\mathrm{b}_{\mathrm{i}}^{2 \mathrm{k}}\right)-\mathrm{a}_{\mathrm{i}}^{4} \sum_{\mathrm{k}=0}^{\infty} \beta_{\mathrm{k}}\left(\mathrm{b}_{\mathrm{i}}^{2 \mathrm{k}}\right)\right]
$$

and the constants $a_{i}$ and $b_{i}$ are given by

$$
a_{i}=\frac{d_{A}}{L-d_{0}-(i-2) d_{A}} \quad b_{i}=\frac{L-d_{0}-(i-1) d_{A}}{L-d_{0}-(i-2) d_{A}}
$$

The maximum number of molecules of diameter $d(\AA)$ that can be accommodated with their centers along the circumference of a circle of diameter $D$ is given by $N=$ $\operatorname{int}\left[\pi / \operatorname{Sin}^{-1}(d / D)\right]$ for $d \leq D$ and $N=1$ for $d>D$. For the $i^{\text {th }}$ layer of adsorbate molecules within the pore, the molecules lie along a circle of diameter $2\left[L-d_{0}-(i-1) d_{A}\right]$, and, hence, the maximum number of molecules of diameter $d_{A}$ that are present in a horizontal cross-section of the $i^{\text {th }}$ layer (when $d_{A} \leq 2\left[L-d_{0}-(i-1) d_{A}\right.$ ) can be written as

$$
N_{i}=\frac{\pi}{\sin ^{-1}\left[\frac{d_{A}}{2\left(L-d_{0}-(i-1) d_{A}\right)}\right]}
$$

However, if $d_{A}>2\left[L-d_{0}-(i-1) d_{A}\right], N_{i}=1$.

Thus, the average interaction energy $\bar{\epsilon}$ can now be written down by a population-weighted average as follows

$$
\bar{\epsilon}=\frac{\sum_{i=1}^{M} N_{i} \epsilon_{i}}{\sum_{i=1}^{M} N_{i}}
$$

The PSD for the cylindrical pore model can now be easily determined using either the HK Eq. 19, or the HK-CY Eq. 20. The algorithm for determining pore-size using the cor-
Table 3. Algorithm (Pseudo-C ode) for O btaining Effective Pore Diameter of Cylindrical Pores

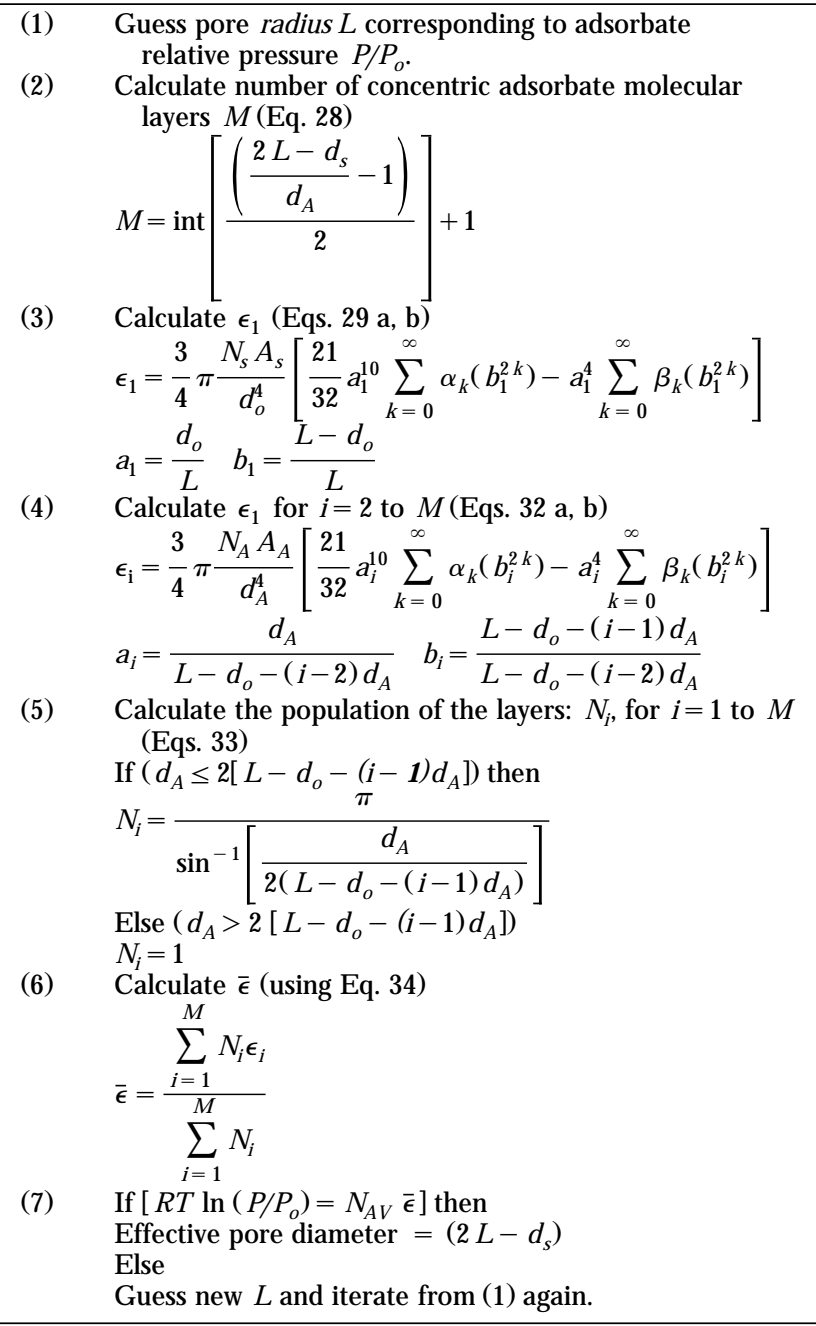

rected $\mathrm{HK}$ equation for cylindrical pores is outlined in Table 3.

\section{Review of Original HK Model for Spherical Pores}

The interaction between an adsorbate molecule and a single layer of sorbent molecules located along the wall of a spherical cavity has been studied in detail in literature (Walker, 1966; Soto et al., 1981; Baksh and $Y$ ang, 1991). If L is the radius of the cavity, $r$ is the radial distance of a gas molecule from the center of the cavity, and $\mathrm{N}$ is the number of molecules on the enveloping cavity surface, the energy potential $\epsilon(r)$ is given as

$$
\begin{aligned}
\epsilon(r)= & 2 N \epsilon_{12}^{*}\left[-\left(\frac{d_{0}}{L}\right)^{6} \frac{1}{4\left(\frac{r}{L}\right)}\left(\frac{1}{\left(1-\frac{r}{L}\right)^{4}}-\frac{1}{\left(1+\frac{r}{L}\right)^{4}}\right)\right. \\
& \left.+\left(\frac{d_{0}}{L}\right)^{12} \frac{1}{10\left(\frac{r}{L}\right)}\left(\frac{1}{\left(1-\frac{r}{L}\right)^{10}}-\frac{1}{\left(1+\frac{r}{L}\right)^{10}}\right)\right]
\end{aligned}
$$


U sing the same approach of Horváth and Kawazoe (1983), the potential expression after taking into account the adsorbate-adsorbate interaction results in the following form

$$
\begin{aligned}
& \epsilon(r)= 2\left(N_{1} \epsilon_{12}^{*}+N_{2} \epsilon_{22}^{*}\right)\left[-\left(\frac{d_{0}}{L}\right)^{6} \frac{1}{4\left(\frac{r}{L}\right)}\right. \\
& \times\left(\frac{1}{\left(1-\frac{r}{L}\right)^{4}}-\frac{1}{\left(1+\frac{r}{L}\right)^{4}}\right)+\left(\frac{d_{0}}{L}\right)^{12} \frac{1}{10\left(\frac{r}{L}\right)} \\
&\left.\left(\frac{1}{\left(1-\frac{r}{L}\right)^{10}}-\frac{1}{\left(1+\frac{r}{L}\right)^{10}}\right)\right] \\
& N_{1}=4 \pi L^{2} N_{s}
\end{aligned}
$$

where

$$
\mathrm{N}_{2}=4 \pi\left(\mathrm{L}-\mathrm{d}_{0}\right)^{2} \mathrm{~N}_{\mathrm{A}}
$$

and the minimum energies are given as

$$
\begin{aligned}
& \epsilon_{12}^{*}=\frac{A_{S}}{4 d_{0}^{6}} \\
& \epsilon_{22}^{*}=\frac{A_{A}}{4 d_{A}^{6}}
\end{aligned}
$$

By integrating over the volume of the spherical cavity, Cheng and $Y$ ang (1994) arrived at the following $\mathrm{HK}$ equation for spherical pores

$$
\begin{aligned}
& R T \ln \left(\frac{P}{P_{0}}\right)=N_{A \nu} \frac{6\left(N_{1} \epsilon_{12}^{*}+N_{2} \epsilon_{22}^{*}\right) L^{3}}{\left(L-d_{0}\right)^{3}} \\
& \times\left[-\left(\frac{d_{0}}{L}\right)^{6}\left(\frac{1}{12} T_{1}-\frac{1}{8} T_{2}\right)+\left(\frac{d_{0}}{L}\right)^{12}\left(\frac{1}{90} T_{3}-\frac{1}{80} T_{4}\right)\right],
\end{aligned}
$$

where

$$
\begin{aligned}
& T_{1}=\frac{1}{\left(1-\frac{L-d_{0}}{L}\right)^{3}}-\frac{1}{\left(1+\frac{L-d_{0}}{L}\right)^{3}} \\
& T_{2}=\frac{1}{\left(1+\frac{L-d_{0}}{L}\right)^{2}}-\frac{1}{\left(1-\frac{L-d_{0}}{L}\right)^{2}} \\
& T_{3}=\frac{1}{\left(1-\frac{L-d_{0}}{L}\right)^{9}}-\frac{1}{\left(1+\frac{L-d_{0}}{L}\right)^{9}} \\
& T_{4}=\frac{1}{\left(1+\frac{L-d_{0}}{L}\right)^{8}}-\frac{1}{\left(1-\frac{L-d_{0}}{L}\right)^{8}}
\end{aligned}
$$

On applying the Cheng-Y ang correction for nonlinearity of the isotherm, the following original $\mathrm{HK}-\mathrm{CY}$ model is obtained

$$
\begin{array}{r}
\mathrm{RT} \ln \left(\frac{\mathrm{P}}{\mathrm{P}_{\mathrm{o}}}\right)-\mathrm{RT}\left[1-\frac{1}{\theta} \ln (1-\theta)\right] \\
=\mathrm{N}_{\mathrm{A} \nu} \frac{6\left(\mathrm{~N}_{1} \epsilon_{12}^{*}+\mathrm{N}_{2} \epsilon_{22}^{*}\right) \mathrm{L}^{3}}{\left(\mathrm{~L}-\mathrm{d}_{\mathrm{o}}\right)^{3}}\left[-\left(\frac{\mathrm{d}_{0}}{\mathrm{~L}}\right)^{6}\left(\frac{1}{12} \mathrm{~T}_{1}-\frac{1}{8} \mathrm{~T}_{2}\right)\right. \\
\left.+\left(\frac{\mathrm{d}_{\mathrm{o}}}{\mathrm{L}}\right)^{12}\left(\frac{1}{90} \mathrm{~T}_{3}-\frac{1}{80} \mathrm{~T}_{4}\right)\right],
\end{array}
$$

where $T_{1}-T_{4}$ are given by $E$ qs. $42-45$. A gain, as in the previous two models, there is room for improving the adsorbate-adsorbate interaction estimate by considering a more physically realistic picture of the molecular distribution in the pore.

\section{Modified HK Model for Spherical Pores}

The modified HK model for a spherical pore follows essentially the same logic as that for slit and cylindrical shaped pores. The total number of adsorbate layers in the form of spherical shells that can be accommodated within a pore of radius $L$ is determined. The energy potential of each gas molecule is computed considering the interaction with the immediately enveloping spherical shell of molecules, which would be the sorbent in the case of the first shell, or the sorbate in the case of subsequent shells. As before, the interaction of the sorbate molecule with the molecules in its own layer, as well as with those in the layers in the interior of its shell, is neglected.

Similar to the cylindrical model, the number of adsorbate layers ( $M$ ) that can be accommodated with a spherical pore of radius $L$, is given by

$$
M=\operatorname{int}\left[\frac{\left(\frac{2 L-d_{s}}{d_{A}}-1\right)}{2}\right]+1
$$

The first layer of adsorbate molecules in the spherical cavity is enclosed by the sorbent molecules along the cavity wall of radius $\mathrm{L}$. The molecules have a maximum probability of existing at a position of minimum potential, which corresponds to a radius of $\left(L-d_{0}\right)$ from the center. $U$ sing an expression similar to $\mathrm{Eq}$. 33, the energy potential of the first layer can thus be written as

$$
\begin{aligned}
\epsilon_{1}=2 N_{0} \epsilon_{12}^{*}\left[-\frac{C_{1}^{6}}{4 f_{1}}\right. & \left(\frac{1}{\left(1-f_{1}\right)^{4}}-\frac{1}{\left(1+f_{1}\right)^{4}}\right) \\
& \left.+\frac{C_{1}^{12}}{10 f_{1}}\left(\frac{1}{\left(1-f_{1}\right)^{10}}-\frac{1}{\left(1+f_{1}\right)^{10}}\right)\right]
\end{aligned}
$$

where $\mathrm{N}_{0}$, the total number of sorbent molecules in the cavity wall single lattice plane, can be found from the density 
(based on area) as follows

$$
\mathrm{N}_{0}=4 \pi \mathrm{L}^{2} \mathrm{~N}_{\mathrm{s}}
$$

Note that since an adsorbate-adsorbent interaction is involved, the depth of energy potential is $\epsilon_{12}^{*}$. The constant $c_{1}$ (and, in general, $c_{i}$ ) is the ratio of the internuclear distance between the interacting molecules under consideration and the radius of the outer enclosing shell of molecules, whereas $f_{1}$ (and, in general, $f_{i}$ ) is the ratio of the radius of the layer of molecules for which the energy potential is being calculated to the radius of the outer enclosing shell of molecules. For the first layer, therefore, these constants are given as

$$
c_{1}=\frac{d_{0}}{L} \quad f_{1}=\frac{L-d_{0}}{L}
$$

The number of molecules existing in the first spherical layer of sorbate molecules can be estimated as

$$
\mathrm{N}_{1}=4 \pi\left(\mathrm{L}-\mathrm{d}_{0}\right)^{2} \mathrm{~N}_{\mathrm{A}}
$$

The second layer of molecules exists at a radius of $\left(L-d_{0}-\right.$ $d_{A}$ ) will be enclosed by the first layer of sorbate molecules at a radius of $\left(L-d_{0}\right)$. Also, since now an adsorbate-adsorbate interaction is involved, the depth of energy potential is $\epsilon_{22}^{*}$. The energy of interaction can be written as

$$
\begin{aligned}
\epsilon_{2}=2 N_{1} \epsilon_{22}^{*}\left[-\frac{C_{2}^{6}}{4 f_{2}}\right. & \left(\frac{1}{\left(1-f_{2}\right)^{4}}-\frac{1}{\left(1+f_{2}\right)^{4}}\right) \\
& \left.+\frac{C_{2}^{12}}{10 f_{2}}\left(\frac{1}{\left(1-f_{2}\right)^{10}}-\frac{1}{\left(1+f_{2}\right)^{10}}\right)\right]
\end{aligned}
$$

and the constants $c_{2}$ and $f_{2}$ are given as

$$
c_{2}=\frac{d_{A}}{L-d_{0}} \quad f_{2}=\frac{L-d_{0}-d_{A}}{L-d_{0}}
$$

The number of molecules in the second layer is

$$
\mathrm{N}_{2}=4 \pi\left(\mathrm{L}-\mathrm{d}_{0}-\mathrm{d}_{\mathrm{A}}\right)^{2} \mathrm{~N}_{\mathrm{A}}
$$

Likewise, the third layer of molecules is situated at a radial distance of $\left(L-d_{0}-2 d_{A}\right)$ enclosed by an adsorbate layer of radius $\left(L-d_{0}-d_{A}\right)$. Thus, its energy potential would be

$$
\begin{aligned}
\epsilon_{3}=2 N_{2} \epsilon_{22}^{*}\left[-\frac{C_{3}^{6}}{4 f_{3}}\right. & \left(\frac{1}{\left(1-f_{3}\right)^{4}}-\frac{1}{\left(1+f_{3}\right)^{4}}\right) \\
& \left.+\frac{C_{3}^{12}}{10 f_{3}}\left(\frac{1}{\left(1-f_{3}\right)^{10}}-\frac{1}{\left(1+f_{3}\right)^{10}}\right)\right]
\end{aligned}
$$

The constants therein as well as the population of molecules inhabiting the third layer can be expressed as

$$
\begin{gathered}
C_{3}=\frac{d_{A}}{L-d_{0}-d_{A}} \quad f_{3}=\frac{L-d_{0}-2 d_{A}}{L-d_{0}-d_{A}} . \\
N_{3}=4 \pi\left(L-d_{0}-2 d_{A}\right)^{2} N_{A}
\end{gathered}
$$

By an inspection of the above equations, one can write down a generalized equation for the ith layer $(i>1)$ of sorbate molecules as follows

$$
\begin{array}{r}
\epsilon_{i}=2 N_{i-1} \epsilon_{22}^{*}\left[-\frac{C_{i}^{6}}{4 f_{i}}\left(\frac{1}{\left(1-f_{i}\right)^{4}}-\frac{1}{\left(1+f_{i}\right)^{4}}\right)\right. \\
\left.\quad+\frac{c_{i}^{12}}{10 f_{i}}\left(\frac{1}{\left(1-f_{i}\right)^{10}}-\frac{1}{\left(1+f_{i}\right)^{10}}\right)\right] \\
C_{i}=\frac{d_{A}}{L-d_{0}-(i-2) d_{A}} f_{i}=\frac{L-d_{0}-(i-1) d_{A}}{L-d_{0}-(i-2) d_{A}} \\
N_{i-1}=4 \pi\left[L-d_{0}-(i-2) d_{A}\right]^{2} N_{A}
\end{array}
$$

As before, the average interaction energy of the adsorbate molecule in the pore would be given by a population-weighted average of the energy potentials of individual molecule layers

$$
\bar{\epsilon}=\frac{\sum_{i=1}^{M} N_{i} \epsilon_{i}}{\sum_{i=1}^{M} N_{i}}
$$

where $M$, the number of adsorbate layers present in the filled spherical pore, is estimated using $\mathrm{Eq}$. 47. $\epsilon_{\mathrm{i}}$ and $\mathrm{N}_{\mathrm{i}}$ are given by $E$ qs. 48 and $58-61$. The HK model (Eq. 19) or the HK-CY model (Eq. 20) can be now used to process the isotherm data in order to obtain the PSD. The algorithm for determining pore size using the corrected $\mathrm{HK}$ equation for spherical pores is given in Table 4 .

In the subsequent sections, the new models would be compared vis-à-vis the original $\mathrm{HK}$ and $\mathrm{HK}-\mathrm{CY}$ equations using isotherm data reported in literature.

\section{Results and Discussion}

In this section, the corrected $\mathrm{HK}$ model will be compared against the original $\mathrm{HK}$ model by studying the profiles of the average interaction energy variation with pore size, as well as by obtaining the PSD from the isotherm data reported in literature. M ost of the examples considered in this work have been studied earlier by Chen and Y ang (1994). The parameters used in the models have been summarized in Table 1 . It should be noted that there are a few differences in the parameters used in this work and the previous work by Cheng and $Y$ ang (1994). A primary difference is the size of the argon molecule. A study of the literature employing the $\mathrm{H}$ orváth-Kawazoe equation showed that there is a wide disparity in the size of the argon molecule used ranging from $2.89 \AA$ (V enero and Chiou, 1988) to $3.36 \AA$ (Cheng and $Y$ ang, 1994). The value of the adsorbate molecular diameter chosen in past literature was actually a "fitting" parameter in order to obtain a better fit to the actual PSD of a material. In this work, however, an A r molecular diameter of $3.82 \AA$ has been used in most cases in this work which is also the more accepted value in literature (Breck, 1974; Razmus and $\mathrm{H}$ all, 1991). However, for the PSD of faujasite using the slit-pore model, an Ar value of $2.95 \AA$ was found to be more appropri- 
Table 4. Algorithm (Pseudo-C ode) for Obtaining Effective Pore Diameter of Spherical Pores

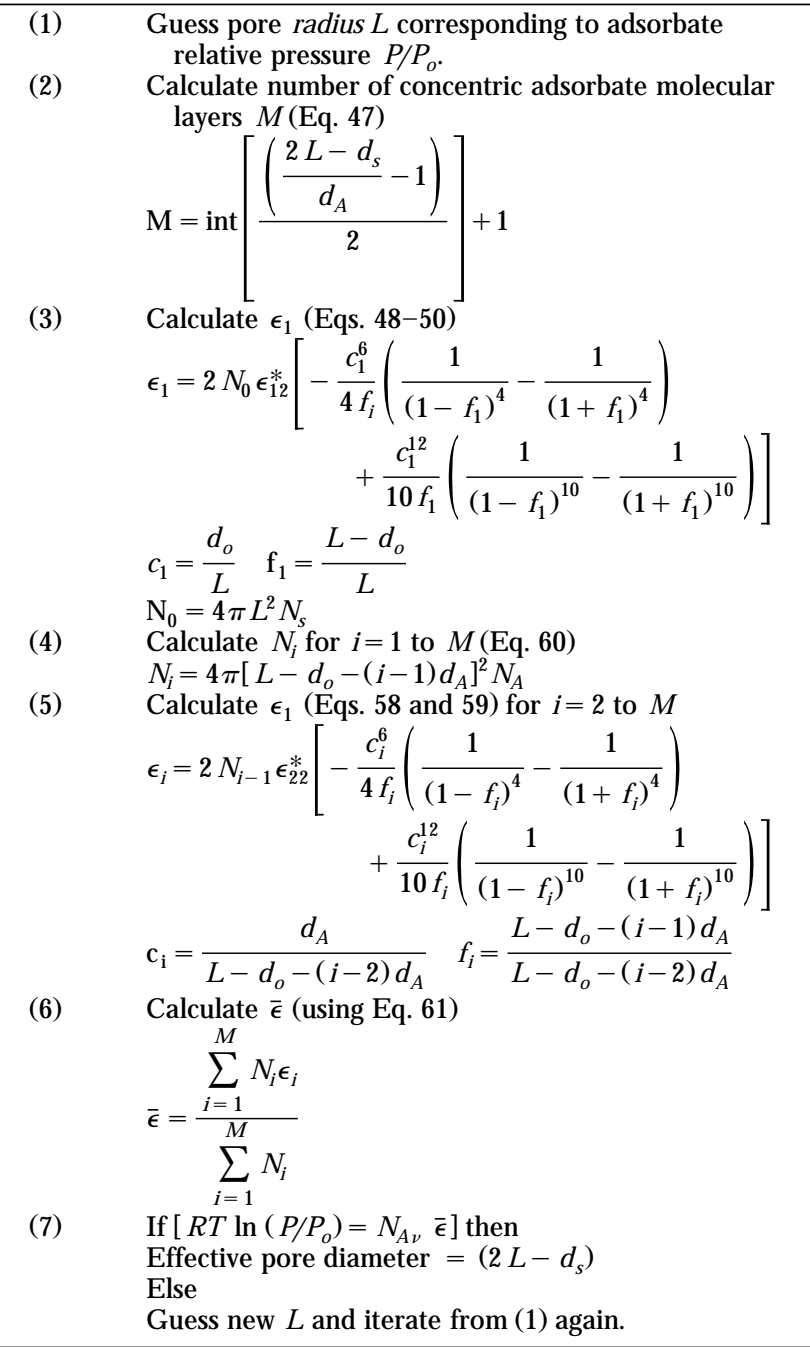

ate. Also, there is a minor difference in the oxide ion surface density value for aluminophosphate materials. The value of $1.48 \times 10^{15}$ molecules $/ \mathrm{cm}^{2}$ used by Cheng and $Y$ ang (1994) was arrived at by assuming a closed packed arrangement of oxide ions of diameter 2.6 A. A study of the structure of aluminophosphates such as $\mathrm{AIPO}_{4}-5$ and $\mathrm{VPI}-5$ revealed, however, that, assuming an $A_{0} L-O$ bond length of $1.73 \mathrm{~A}$ and $a$ P-O bond length of $1.66 \AA$, the average oxide ion density on the cylindrical channel surface was closer to $1.0 \times 10^{15}$ molecules $/ \mathrm{cm}^{2}$. Choosing the right physical parameters is critical for discriminating between $\mathrm{H}$ orváth-K awazoe models, since the predicted pore size is known to be very sensitive to small variations in the physical parameters used (Saito and Foley, 1991).

\section{Slit-shaped pore model}

HGS-638 Molecular Sieve Carbon. The modified energy profile model developed in this work was compared vis-á-vis the original HK model. A s a model system, adsorption of liquid nitrogen at $77 \mathrm{~K}$ on HGS 638 molecular sieve carbon

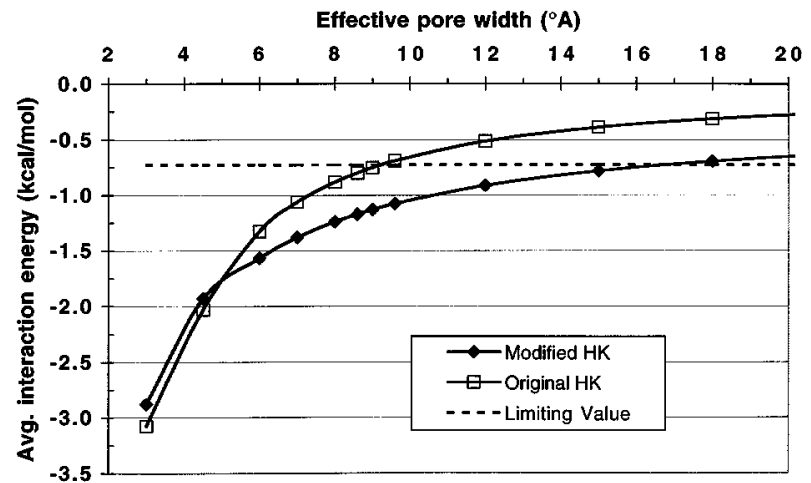

Figure 6. Variation of average interaction energy of $\mathrm{N}_{2}$ molecules vs. pore size of molecular sieve carbon as predicted by original and modified HK models for slit-shaped pores.

sorbent was considered which was the same system studied by Horváth and Kawazoe (1983). The physical parameters used in the model are summarized in Table 1. The comparison of the average interaction energy with respect to the slit pore size expressed in multiples of the $\mathrm{N}_{2}$ molecular diameter $(3.0 \mathrm{~A})$ is shown in Figure 6 . From the figure, it can be inferred that the average interaction energy predicted by both the models is approximately the same for pore widths measuring 1 to 2 times the adsorbate molecular diameter. This is expected since the sorbate-sorbate interaction has a negligible contribution compared to the overlapping sorbate-sorbent energy potentials at very small pore sizes. $\mathrm{H}$ owever, beyond a pore size of $2 d_{A}$, the modified model predicts a much lower energy potential than the original model. Thus, the pore size predicted by using the original $\mathrm{HK}$ model would be an underestimate of the actual pore size for pore widths exceeding $2 d_{A}$. A s was explained earlier, the system should tend to have a limiting maximum interaction energy equal to that given by Eq. 11. A Fit of Eq. 10 with the N2/HGS-638 M SC data given in the study by $\mathrm{H}$ orváth and Kawazoe (1983) gave a value of $K=175$. Substituting this $K$ value in the righthand side of $\mathrm{Eq}$. 11 gives a limiting value of $-0.73 \mathrm{kcal} / \mathrm{mol}$. This should be the limiting magnitude of interaction energy in a pore of infinite width and is indicated as dotted line in Figure 6. It was interesting to note that while the interaction energy predicted by the original model tends towards zero at large pore widths, that predicted by the proposed model tends to a value closer to the limiting interaction energy. An inspection of $E$ q. 15 reveals that as $L \rightarrow \infty$ (that is, $M \rightarrow \infty$ ), the average interaction energy tends to $\mathrm{N}_{\mathrm{A} v} \epsilon_{3}(\mathrm{z})$. For the $\mathrm{N}_{2} / \mathrm{HGS}-638$ system, this limiting value was determined to be $-0.3 \mathrm{kcal} / \mathrm{mol}$. The two values are remarkably similar considering the fact that the limiting value given by $\mathrm{Eq} .10$ is purely from experimental isotherm data while that obtained from Eqs. 14 and 15 are purely from Lennard-J ones interaction parameters. Besides, the error is also likely to be caused due to an assumption in the $\mathrm{HK}$ derivation that the entropy of adsorption can be neglected compared to the enthalpy of adsorption, whereas the entropy change can be considerable at large pore sizes.

The PSD of HGS-638 predicted by the original HK Eq. 8, original HK-CY Eq. 9, modified-HK Eq. 19 and the modified 


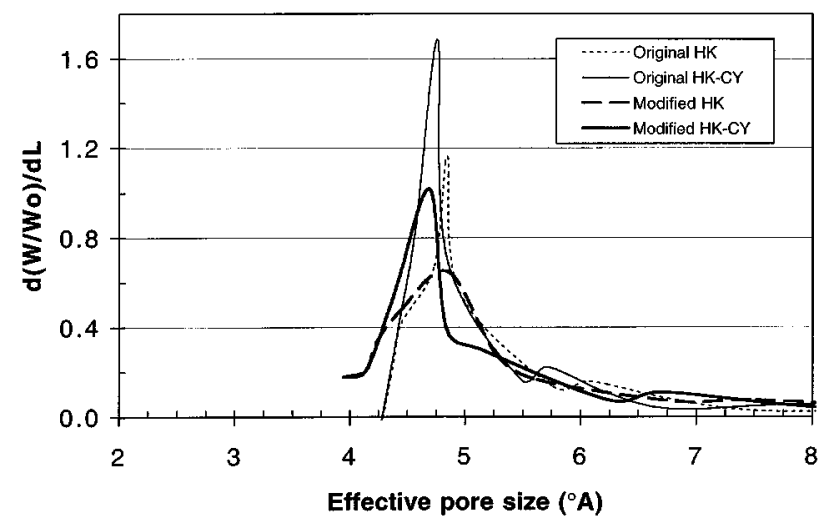

Figure 7. PSD of HGS-638 molecular sieve carbon as predicted by original and modified HK models for slit-shaped pores.

$\mathrm{HK}-\mathrm{CY}$ Eq. 20 is shown in Figure 7. It can be seen that there is virtually no shift in the peak pore size when the interaction energy model was changed. This is so because the peak pore size of $\mathrm{HGS}-638$ lies in the $4-6^{\circ} \mathrm{A}$ range, which corresponds to roughly twice the adsorbate molecule $\left(\mathrm{N}_{2}\right)$ diameter. It is evident from Figure 6 that the interaction energy predicted by the two models is nearly the same until the slit width is about $2 d_{A}$ and, hence, the predicted pore widths are also similar in this size range. A lso, it was observed that the points on the figure corresponding to the higher extreme of the pore width in the original model appeared at a still higher pore size in the new model. This again can be explained from the fact that, for the same interaction energy, the new model predicts a higher pore size compared to that predicted by the original model.

\section{F aujasite zeolite}

The adsorption isotherm of argon on faujasite zeolite at 87 $K$ has been given by Borghard et al. (1991). This type of zeolite actually has a spherical cavity and the results using the modified HK model for spherical pores will be subsequently shown. The slit-pore model was tried in the case of faujasite,

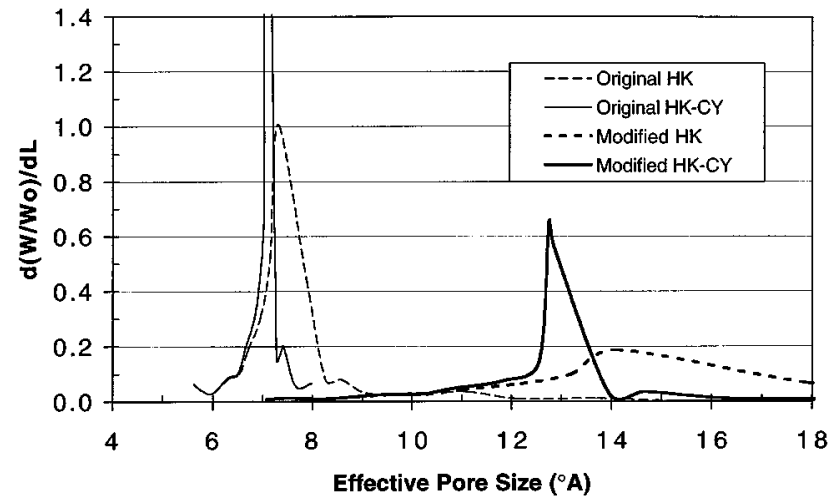

Figure 8. PSD of faujasite zeolite as predicted by original and modified HK models for slit-shaped pores. particularly since it has a comparatively larger pore structure than molecular sieve carbons and the differences between the model predictions will become obvious. It has been shown previously (Cheng and $Y$ ang, 1994) that the application of the original HK model gives a highly underestimated PSD. Figure 8 shows the prediction of the two original and two modified models. It can be seen that while the original models predicted a pore size of $7 \AA$, the modified models gave a peak at much higher pore size of $13 \mathrm{~A}$. It is interesting to note that the crystallographic channel size of $F$ aujasite is 7.4 $\AA$, while the actual cavity size of Faujasite is $13.7 \AA$. Thus, the modified model is seen to be more capable of determining the PSD in the higher range of pore size compared to the original model. This is a result of a more realistic picture for calculation of adsorbate-adsorbate interaction and an improved method of averaging interaction energy.

\section{Cylindrical Pore Model ZSM-5 zeolite}

The zeolite ZSM - 5 is an aluminosilicate with three-dimensional channels measuring $5.6 \times 5.3 \AA$ in width. The isothermal of argon on ZSM -5 zeolite at $87 \mathrm{~K}$ has been reported by $V$ enero and Chiou (1988). Since the channels for this adsorbent are approximately cylindrical in nature, the cylindershaped pore model would be ideal for this example. Figure 9 shows the average interaction energy of an argon molecule at $87 \mathrm{~K}$ in ZSM -5 pore filled with adsorbate molecules at different pore diameters, as predicted by the original and modified $\mathrm{HK}$ cylindrical pore models. It can be seen that although the average energy within the pore predicted by the two models was reasonably close at low pore sizes $(<7 \AA)$, the difference became more pronounced in the higher size range. A fit of the isotherm data to $E q .10$ gave a value of $K=770$. $U$ sing this value of $K$, a limiting interaction energy value of -0.98 $\mathrm{kcal} / \mathrm{mol}$ was obtained at large pore sizes ( $\mathrm{Eq}$. 11), which is depicted in the form of a dotted line in Figure 9. From the prediction using the Lennard-J ones parameters, it can be seen that at large pore sizes, a limiting value of $-0.70 \mathrm{kcal} / \mathrm{mol}$ is obtained. The reasonably good agreement of the two values,

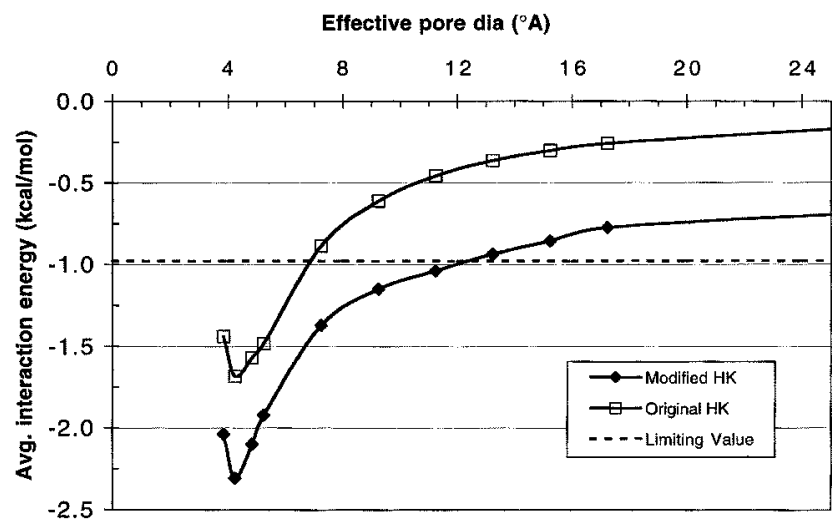

Figure 9. Variation of average interaction energy of argon molecules vs. pore size of ZSM-5 zeolite as predicted by original and modified HK models for cylindrical pores. 


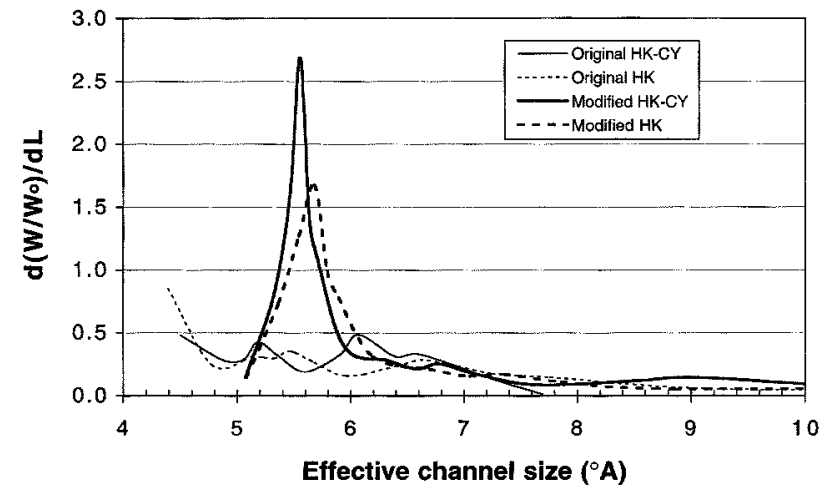

Figure 10. PSD of ZSM-5 zeolite as predicted by original and modified HK models for cylindrical pores.

one from purely empirical data and the other from established interaction parameters, adds to the merit of the modified model.

The PSD of ZSM -5 predicted by the four models is shown in Figure 10. It was seen that for the parameters chosen ( $\mathrm{Ta}$ ble 1), no prominent peak was visible for both the original $\mathrm{HK}$, as well as the $\mathrm{HK}-\mathrm{CY}$ model since no solution for the pore size existed for the first few points of the isotherm. On the other hand, a peak was obtained at $5.7 \AA$ using the modified $\mathrm{HK}$ model. On using the modified $\mathrm{HK}-\mathrm{CY}$ model, a sharper peak was observed at $5.6 \AA$, which agrees well with the crystallographic dimension of the ZSM -5 channel.

\section{MCM-41 molecular sieves}

MCM-41 materials belong to the family of large pore (mesoporous) molecular sieves and are increasingly becoming popular as catalyst supports. These synthetic materials possess a regular array of hexagonal, uniform unidimensional mesopores, which can be systematically varied from 16-100 $A$. Due to their narrow PSD, minimal network effects, and well-known surface chemistry, they are ideal candidates for testing pore-size models. Numerous studies have been conducted for studying the PSD of M CM - 41 materials using various techniques such as the $\mathrm{BJH}$ method (R avikovitch et al., 1997; Sayari et al., 1999), X-ray diffraction (XRD) (Ravikovitch et al., 1995), H-N MR (Schmidt et al., 1995), and nonlocal density functional theory (NLDFT) (Ravikovitch et al., 1995, 1997).

In order to test the corrected $\mathrm{HK}$ model, $\mathrm{N}_{2}$ adsorption data at $77.4 \mathrm{~K}$ on a M CM-41 material denoted "Sample C" in a previously published work (R avikovitch et al., 1995) was used. The XRD results and the NLDFT simulation studies reported in the latter work show the pore size of the material to be $45 \AA$. Figure 11 shows the PSD for the M CM - 41 material predicted from the $\mathrm{N}_{2}$ adsorption data using the corrected and original $\mathrm{HK}$ cylindrical pore models. Since the isotherm does not follow Langmuirian behavior, the Cheng$Y$ ang correction was computed by fitting the data to a Langmuir isotherm as much as possible. The figure shows that the corrected $\mathrm{HK}-\mathrm{CY}$ equation accurately estimates the pore size of the material to be $45 \AA$. U sing the same model parameters

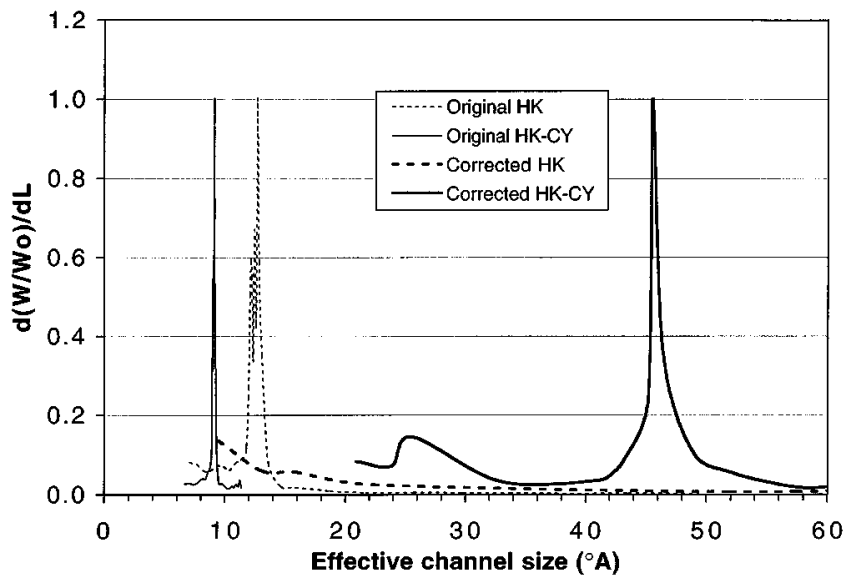

Figure 11. PSD of MCM-41 material as predicted by original and modified HK models for cylindrical pores.

(shown in Table 1), the original $\mathrm{HK}$ and $\mathrm{HK}-\mathrm{CY}$ models give a grossly underestimated pore size of $12 \AA$ and $9 \AA$, respectively. It must be noted that the corrected $\mathrm{HK}$ equation without the Cheng-Y ang correction gives a highly overestimated value of pore size (beyond the limits of Figure 11). This is because the inflexion on the curve occurs at a high $\mathrm{P} / \mathrm{P}_{\mathrm{s}}$ value ( $\sim 0.4$ ), and there is a severe deviation from Henry's law at this value. Further, R avikovitch et al. (1997) have reported that the $\mathrm{BJH}$ method systematically underestimated the pore size of the MCM-41 materials studies by $\sim 10 \mathrm{~A}$. Thus, the utility of the corrected $\mathrm{HK}-\mathrm{CY}$ model in predicting PSD for materials in the mesoporous ( $>20 \mathrm{~A}$ ) size range is demonstrated.

\section{$\mathrm{AlPO}_{4}$ (Aluminophosphates)}

Like zeolitic materials, aluminophosphates also poses well-defined structure and narrow PSD. M ost aluminophosphates such as $\mathrm{AIPO}_{4}-11$ and $\mathrm{VPI}-5$ have a one-dimensional channel structure. Adsorption isotherms of argon on these materials have been reported by $D$ avis et al. (1988).

The PSD of $\mathrm{AIPO}_{4}-11$ is shown in Figure 12. The actual channel size reported in literature is $6.3 \AA$ ( $M$ eier and O Ison,

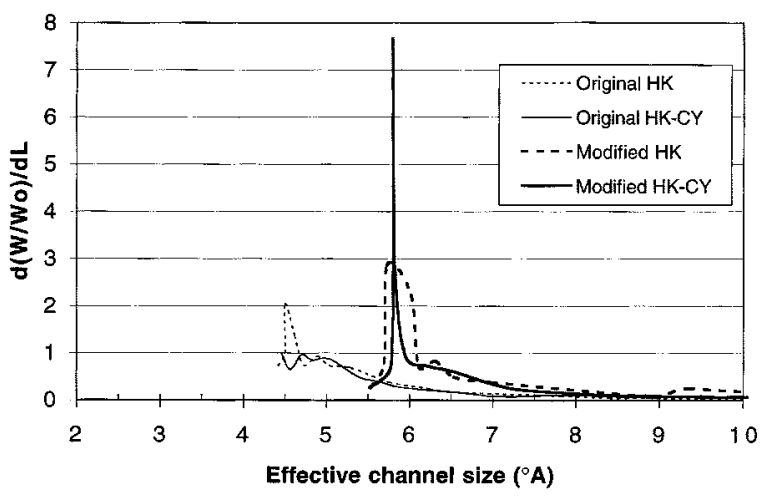

Figure 12. PSD of AIPO $_{4}-11$ as predicted by original and modified HK models for cylindrical pores. 


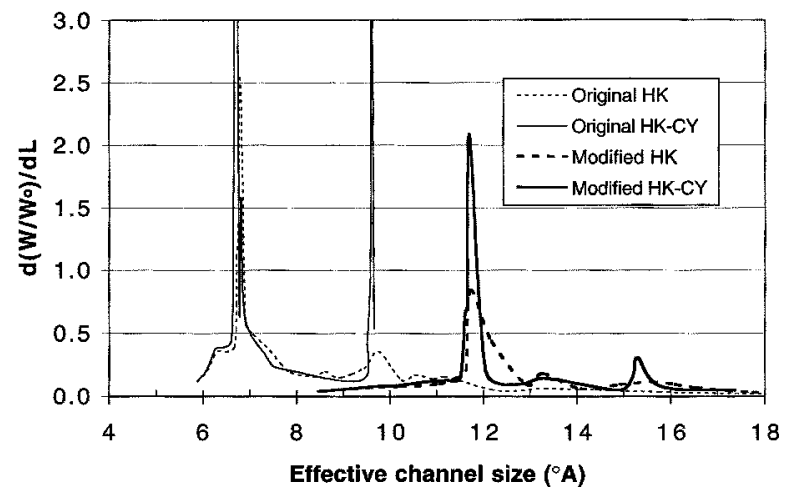

Figure 13. PSD of VPI-5 as predicted by original and modified HK models for cylindrical pores.

1987). The modified HK-CY model gives a peak at a pore size of about $5.9 \AA$. The original model on the other hand gives a peak at a much lower pore size at $4.6 \AA$. Figure 13 shows the computed PSD of V PI-5 aluminophosphate, whose isotherm is seen to have two sigmoidal bends suggesting that it may have a bimodal PSD. The currently accepted pore size of $\mathrm{VPI}-5$ is $12.1 \AA$ (Davis et al., 1988). It was shown previously by Cheng and $\mathrm{Y}$ ang (1994) that V PI-5 gives two peaks on employing the original $\mathrm{HK}-\mathrm{C}_{0}$ equation: a major peak at 8.6 $\mathrm{A}$ and a minor one at $11.7 \mathrm{~A}$. In this work different parameters were used for the kinetic diameter of argon and the oxide ion density in the aluminophosphate framework. As a result, the new peaks obtained using the original model were situated at $6.8 \AA$ and at $8.7 \AA$. With the modified $\mathrm{HK}-\mathrm{CY}$ model, the major peak was observed at $11.8 \AA$ and a minor peak was observed at $15.2 \AA$. Thus, the modified cylindrical $\mathrm{HK}-\mathrm{CY}$ model has a better agreement with crystallographic data than the original model.

\section{Spherical Pore Model F aujasite zeolite}

The PSD for faujasite zeolite using the slit pore model was shown in an earlier section. This zeolite is, however, known to have nearly spherical shaped cavities measuring $13.7 \AA$ in diameter. $\mathrm{H}$ ence, it is apparent that the spherical pore model would be more appropriate to obtain the PSD for this material. The average interaction energy of an A molecule in an adsorbate filled Faujasite micropore predicted by the original and modified $\mathrm{HK}$ equations is shown in Figure 14. It can be seen from the figure that the predicted average energy potentials for both the models are nearly the same until an effective pore size of $8 \AA$. A t higher pore diameters, a consider able difference in calculated average energy values was observed. As in previous examples of models with other pore geometries, the interaction energy calculated by the original HK model was seen to approach zero at large pore size, whereas that predicted by the new model was seen to asymptotically approach a value of about $-0.75 \mathrm{kcal} / \mathrm{mol}$. A fit of the isotherm data to $E$ q. 9 gave a value of $K=920$, which on substitution in $\mathrm{Eq}$. 10 resulted in a limiting value of -1.0 $\mathrm{kcal} / \mathrm{mol}$ of the interaction energy (shown as a dotted line in the figure) at large pore size. As before, this is a good agree-

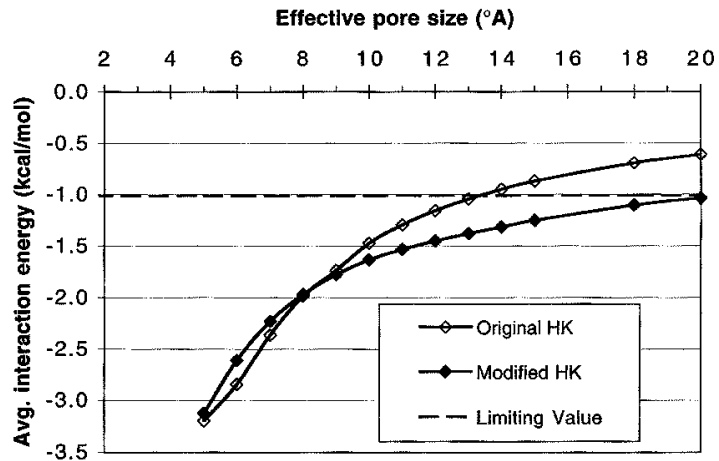

Figure 14. Variation of average interaction energy of argon molecules vs. pore size of faujasite zeolite as predicted by original and HK models for spherical pores.

ment of a theoretical model prediction with an experimentally obtained value.

The spherical-shaped pore model was employed next to deduce the PSD of Faujasite from the argon isotherm data of Borghard et al. (1991). Figure 15 shows the distribution of pores as calculated by the four models. For the parameters chosen, the original HK-CY equation provided a peak at about $10.5 \mathrm{~A}$, whereas the modified $\mathrm{HK}-\mathrm{CY}$ model calculated one at the expected value of $13.7 \mathrm{~A}$. The modified $\mathrm{HK}$ model without the correction for isotherm nonlinearity, however, provides a highly diminished peak at about $14.8 \mathrm{~A}$.

\section{A zeolite}

The adsorption of argon on $5 \mathrm{~A}$ zeolite at $87 \mathrm{~K}$ was measured by Venero and Chiou (1988). On performing a PSD analysis of the isotherm data with the models discussed in this work, it was observed that the original model predicted a pore size of $8.8 A_{0}$, whereas the modified model predicts a pore size of $9.8 \mathrm{~A}$ (Figure 16). In reality, 5A zeolite (also known as ( a-A) has a crystallographic channel size of $4.9 \AA$ (D yer, 1988) and a cavity size of $11.4 \AA$. Thus, the new model provides a reasonably better estimate of the pore size. A gain, the difference in the calculated and the crystallographic value

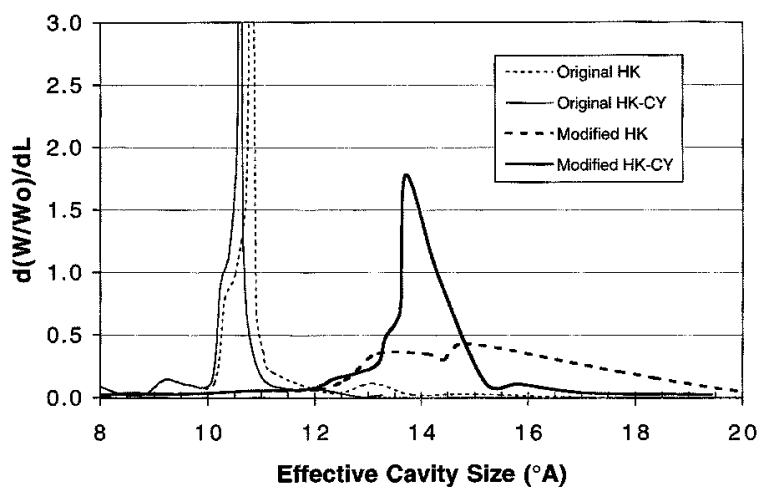

Figure 15. PSD of faujasite zeolite as predicted by original and modified HK models for spherical pores. 




Figure 16. PSD of 5A (Ca-A) zeolite as predicted by original and modified HK models for spherical pores.

can be attributed to the uncertainty in the model parameters used.

\section{Conclusions}

The conventional H orváth-K awazoe equations for calculation of PSD in microporous sorbents have been shown to have certain errors associated in its derivation. The primary causes of error in the original model have been identified as an incorrect method of calculating adsorbate-adsorbate interaction within a filled micropore, an unrealistic picture of distribution of molecules in the micropores, and the lack of inclusion of an energy distribution while calculating the average interaction energy. In this work, new models have been proposed for the purpose of determination of PSD for slit, cylindrical as well as spherical shaped pores. Although the primary principle is the same as that of Horvath and Kawazoe (1983), the proposed model is different with respect to the method of calculation of average interaction energy. A $n$ improved method of calculating adsorbate-adsorbate interaction is demonstrated assuming that molecules occupy positions corresponding to lowest energy potentials. This is in accordance with the Boltzmann law of energy distribution which assigns the highest probability of a molecule to exist in a state for which its energy potential is minimum. The new model is simple to use and can be easily implemented using a programming code or a spreadsheet with a nonlinear regression capability. A study of the average interaction energy vs. pore-size profiles revealed that the original model is reasonably accurate for effective pore sizes measuring less than two adsorbate diameters, but, thereafter, a significant deviation was observed from the modified model. Various examples have been provided for all the three different pore geometries which show that the PSD provided by the corrected model is in better agreement with crystallographic data in literature than the original model. M oreover, the corrected $\mathrm{HK}$ model is shown to accurately predict PSD of even mesoporous materials like M CM -41 materials, which was not possible with the original HK model. Thus, the improved model can be used for the entire nanoporous size range up to the pore size at which the surface tension of the adsorbate in the pore becomes important, in which case the Kelvin equation should be used.

\section{Acknowledgment}

This work was supported by National Science Foundation, Grant CTS-9819008.

\section{Notation}

$a_{i}, b_{i}=$ parameters in modified cylindrical pore model for ith layer of molecules

$\mathrm{c}=$ speed of light $\mathrm{m} / \mathrm{s}$

$c_{i}, f_{i}=$ parameters in modified spherical pore model for ith layer of molecules

$d_{0}=$ mean of the diameters of adsorbate and adsorbent molecules, A

$\mathrm{i}=$ index for sorbate molecular layer numbered starting from outermost layer

$\mathrm{k}=$ summation counter for cylindrical pore energy profile equation, or Boltzmann constant

$\mathrm{K}=$ parameter in the $\mathrm{L}$ angmuir equation

$\mathrm{L}=$ internuclear distance between parallel layers of slitshaped pores, likewise defined as the radius (not diameter) for cylindrical and spherical pore, $A$

$\mathrm{m}=$ mass of an electron, $\mathrm{kg}$

$\mathrm{N}=$ density of molecules based on surface area, (molecules $/ \mathrm{cm}^{2}$ ), or number of molecules in a layer

$\mathrm{N}_{1}=$ number of sorbent molecules on cavity surface

$\mathrm{N}_{2}=$ number of adsorbate molecules in layer

$\mathrm{N}_{\mathrm{Av}}=$ A vogadro's number, molecules $/ \mathrm{mol}$

$\mathrm{P}=$ pressure of adsorbate in the gas phase, bar

$\mathrm{P}_{0}=$ saturation vapor pressure of adsorbate, bar

$\mathrm{r}=$ radial distance of an adsorbate molecule from the central axis for cylindrical pores and from center in case of spherical pores

$\mathrm{R}=$ gas constant, $\mathrm{J} / \mathrm{mol} / \mathrm{K}$

$\mathrm{T}=$ absolute temperature, $\mathrm{K}$

$\alpha=$ polarizability, $\mathrm{cm}^{3}$

$\epsilon_{12}=$ interaction energy potential between molecule 1 and 2, $\mathrm{erg} / \mathrm{molecule}$

$\epsilon_{12}^{*}=$ depth of potential energy minimum for adsorbate-adsorbent interaction, erg/molecule

$\epsilon_{22}^{*}=$ depth of potential energy minimum for adsorbate-adsorbate interaction, erg/molecule

$\bar{\epsilon}=$ average interaction energy, erg

$\theta=$ degree of void filling in the sorbent

$\chi=$ magnetic susceptibility, $\mathrm{cm}^{3}$

\section{Subscripts}

A = corresponding to adsorbate

$\mathrm{S}=$ corresponding to the adsorbent

$\mathrm{A}-\mathrm{S}=$ adsorbate-sorbent interaction

$\mathrm{A}-\mathrm{A}=$ adsorbate-adsorbate interaction

\section{Literature Cited}

A SA P 2010 Operator's M anual, v. 2.00, Micromeritics Instrument Inc., N orcross GA (1995).

Baksh, M. S. A., and R. T. Y ang, "M odel for Spherical Cavity R adii and Potential Functions of Sorbates in Zeolites," AIChE J., 37, 923 (1991).

Baksh, M. S. A., and R. T. Y ang, "U nique Adsorption Properties and Potential Energy Profiles of Microporous Pillared Clay," AIChE J., 38, 1357 (1992).

Barrett, E. P., L. G. J oyner, and P. P. Halenda, "D etermination of Pore Volume and A rea Distributions in Porous Substances, I. Computations from Nitrogen Isotherms," J. Am. Chem. Soc., 73, 373 (1951).

Beck, J. S., J. C. V artuli, W. J. R oth, M. E. Leonowicz, C. T. Kresge, K. D. Schmitt, C. T.-W. Chu, D. H. Olson, E. W. Sheppard, S. B. M cCullen, J. B. Higgins, and J. L. Schlenker, "A New Family of 
M esoporous M olecular Sieves Prepared With Liquid Crystal Templates," J. Am. Chem. Soc., 114, 10835 (1992).

Breck, D. W., Zeolite Molecular Sieves: Structure, Chemistry, and Use, Wiley, N ew Y ork (1974).

Borghard, W. S., E. W. Sheppard, and H. J. Schoennagel, "An A utomated, High Precision U nit for Low-Pressure Physisorption," Rev. Sci. Instrum., 62, 2801 (1991).

Brunauer, S., L. S. Deming, W. S. Deming, and E. Teller, "On Theory of the van der Waals A dsorption of G ases," J. Amer. Chem. Soc., 62, 1723 (1940)

Brunauer, S., R. Sh. Mikhail, and E. E. Bodor, "Pore Structure A nalysis Without a Pore Shape Model," J. Colloid Sci., 24, 451 (1967).

Carrott, P. J. M., M. M. L. R. Carrott, and T. J. M ays, "Comparison of $M$ ethods for $E$ stimating $M$ icropore Sizes in A ctive Carbons from A dsorption I sotherms," Proc. Sixth Intl. Conf. Fundam. of Adsorption, F. M eunier, ed. Elsevier, Paris, 677 (1998).

Cheng, L. S., and R. T. Y ang, "Improved H orváth-K awazoe Equations Including Spherical Pore Models for Calculating Micropore Size D istribution," Chem. Eng. Sci., 49, 2599 (1994).

Davis, M . E., C. M ontes, P. E. H athaway, J. P. A rhancet, D. L. H asha, and J. E. Graces, "Physicochemical Properties of V PI-5" J. Amer Chem. Soc., 111, 3919 (1989).

Davis, M. E., C. Saldarriaga, C. M ontes, J. Garces, and C. Crowder, "VPI-5: The First Molecular Sieve With Pores Larger Than 10 A ngstroms," Zeolites, 8, 362 (1988).

Dubinin, M. M., and L. V. Radushkevich, "The Equations of the Characteristic Curve of A ctivated Charcoal," Doklady A kad. Nauk. S.S.S.R., 55, 327 (1947).

D yer, A ., An Introduction to Zeolite M olecular Sieve, Wiley, N ew Y ork (1988).

Everett, D. H., and J. C. Powl, "A dsorption in Slit-Like and Cylindrical M icropores in the Henry's Law R egion," J. Chem. Soc. Faraday Trans., 72, 619 (1976).

H orváth, G ., V . H alász-L aky, I. D ékány, and F. Berger, "Pore Characterization of Different $A$ dsorbents in M icropore Domain Based on $\mathrm{CO}_{2}$ A dsorption," Proc. Int. Conf. Fundam. of Adsorption, $\mathrm{F}$. M eunier, ed., Elsevier, Paris, 611 (1998).

H orváth, G., and K. Kawazoe, "M ethod for Calculation of Effective Pore Size Distribution in M olecular Sieve Carbon," J. Chem. Eng. Japan, 16, 470 (1983)

Jaroniec, M., and R. M adey, Physical Adsorption on Heterogeneous Solid, Elsevier, A msterdam (1988).

Kane, M. S., J. H. Bushong, H. C. Foley, and W. H. Brendley, "Effect of Nanopore Size Distributions on Trichloroethylene A dsorption and Desorption on Carbogenic A dsorbents," Ind. Eng. Chem. Res., 37, 2416 (1998).

Lastoskie, C., K. E. Gubbins, and N. Quirke, "Pore Size Distribution A nalysis of Microporous Carbons: A Density Functional Theory A pproach,"J. Phys. Chem., 97, 4786, (1993).

M ariwala, R. K., and H. C. Foley, "Calculation of M icropore Sizes in Carbogenic M aterials from M ethyl Chloride A dsorption I sotherm," Ind. Eng. Chem. Res., 33, 2314 (1994).

M eier, W. M . and D. H. Olson, A tlas of Zeolite Structure Types, Butterworth, London (1987)
Olivier, J. P., "M odeling Physical Adsorption on Porous and NonPorous Solids U sing D ensity Functional Theory," J. Porous M aterials, 2, 9 (1995).

Ravikovitch, P. I., D. Wei, G. L. Haller, and A. V. Neimark, "Evaluation of Pore Structure Parameters of M CM-41 Catalyst Supports and Catalysts by $M$ eans of Nitrogen and A rgon A dsorption," J. Phys. Chem. B, 101, 3671 (1997).

R avikovitch, P. I., G. L. H aller, and A . V. N eimark, "A dsorption Characterization of Nanoporous M aterials of M 41S Type," Proc. Sixth Int. Conf. Fundam. of Adsorption, F. M eunier, ed., Elsevier, Paris, 545 (1998)

R avikovitch, P. I,.., S. C. Ó D omhnaill, A . V . N eimark, F. Schüth, and K. K. U nger, "Capillary H ysteresis in Nanopores: Theoretical and Experimental Studies of Nitrogen A dsorption on M CM -41," L angmuir, 11, 4765 (1995).

R azmus, D. M ., and C. K. H all, "Prediction of G as A dsorption in $5 A$ Zeolites U sing M onte Carlo Simulation," AIChE J., 37, 769 (1991).

Saito, A., and H. C. Foley, "Curvature and Parametric Sensitivity in M odels for Adsorption in Micropores," AIChE J., 37, 429 (1991).

Sams, J. R ., J r., G. Constabaris, and G. D. H alsey, "Second V irial Coefficients of Neon, A rgon, Krypton and X enon with a Graphitized Carbon Black," J. Phys. Chem., 64, 1689 (1960).

Schmidt, R., E. W. H ansen, M. Stöcker, D. A kporiaye, and O. H. Ellestad, "Pore Size D etermination of M CM -41 M esoporous M aterials by $M$ eans of $\mathrm{H}-\mathrm{NMR}$ Spectroscopy, $\mathrm{N}_{2}$ Adsorption, and HRE M. A Preliminary Study,"J. Am. Chem. Soc., 117, 4049 (1995).

Seaton, N. A., J. P. R. B. Walton, and N. Quirke, "A N ew A nalysis $M$ ethod for the Determination of the Pore Size Distribution of Porous Carbons from Nitrogen A dsorption M easurements," Carbon, 27, 853 (1989).

Sayari, A ., Y . Y ong, M . Kruk, and M . Jaroniec, "Expanding the Pore Size of MCM-41 Silicas: U se of A mines as Expanders in Direct Synthesis and Postsynthesis Procedures," J. Phys. Chem. B., 103, 3651 (1999).

Seifert, J., and G. E mig, "M ikrostrukturuntersuchungen as Porösen Festsoffen Durch Physisorptionsmessungen," Chem. Eng. Technol., 59, 475 (1987).

Soto, L. J ., P. W. Fisher, A. J . G lessner, and A. L. M yers, "Sorption of Gases in Molecular Sieves-Theory for Henry's Constant," J. Chem. Soc. Faraday Trans., 77, 157 (1981).

Stoeckli, H. F., "A Generalization of the Dubinin-R adushkevich Equation for Filling of $\mathrm{H}$ eterogeneous M icropores," J. Colloid Interface Sci., 59, 184 (1977).

Venero, A. F., and J. N. Chiou, "Characterization of Zeolites by G as Adsorption at Low Pressures," MRS Symp. Proc. 111, 235 (1988).

Walker, P. L., J r., ed., Chemistry and Physics of Carbon, V ol. 2, M arcel Dekker, New Y ork, p. 362 (1966).

W ebb, P. A ., and C. O rr, A nalytical M ethods in Fine Particle Technology, Chap. 3., M icromeritics Inc., N orcross G A (1997).

Y ang, R. T., and M. S. A. Baksh, "Pillared Clay A s a N ew Class of Sorbents for G as Separation," AIChE J., 37, 679 (1991).

Manuscript received July 26, 1999, and revision received N ov. 29, 1999. 